\title{
The dominant Anopheles vectors of human malaria in the Americas: occurrence data, distribution maps and bionomic précis
}

\author{
Marianne E Sinka ${ }^{1 *}$, Yasmin Rubio-Palis ${ }^{2,3}$, Sylvie Manguin ${ }^{4}$, Anand P Patil ${ }^{1}$, Will H Temperley ${ }^{1}$, Peter W Gething ${ }^{1}$, \\ Thomas Van Boeckel ${ }^{1,5}$, Caroline W Kabaria ${ }^{6}$, Ralph E Harbach ${ }^{7}$, Simon I Hay ${ }^{1,6^{*}}$
}

\begin{abstract}
Background: An increasing knowledge of the global risk of malaria shows that the nations of the Americas have the lowest levels of Plasmodium falciparum and P. vivax endemicity worldwide, sustained, in part, by substantive integrated vector control. To help maintain and better target these efforts, knowledge of the contemporary distribution of each of the dominant vector species (DVS) of human malaria is needed, alongside a comprehensive understanding of the ecology and behaviour of each species.

Results: A database of contemporary occurrence data for 41 of the DVS of human malaria was compiled from intensive searches of the formal and informal literature. The results for the nine DVS of the Americas are described in detail here. Nearly 6000 occurrence records were gathered from 25 countries in the region and were complemented by a synthesis of published expert opinion range maps, refined further by a technical advisory group of medical entomologists. A suite of environmental and climate variables of suspected relevance to anopheline ecology were also compiled from open access sources. These three sets of data were then combined to produce predictive species range maps using the Boosted Regression Tree method. The predicted geographic extent for each of the following species (or species complex*) are provided: Anopheles (Nyssorhynchus) albimanus Wiedemann, 1820, An. (Nys.) albitarsis*, An. (Nys.) aquasalis Curry, 1932, An. (Nys.) darlingi Root, 1926, An. (Anopheles) freeborni Aitken, 1939, An. (Nys.) marajoara Galvão \& Damasceno, 1942, An. (Nys.) nuneztovari*, An. (Ano.) pseudopunctipennis* and An. (Ano.) quadrimaculatus Say, 1824. A bionomics review summarising ecology and behaviour relevant to the control of each of these species was also compiled.

Conclusions: The distribution maps and bionomics review should both be considered as a starting point in an ongoing process of (i) describing the distributions of these DVS (since the opportunistic sample of occurrence data assembled can be substantially improved) and (ii) documenting their contemporary bionomics (since intervention and control pressures can act to modify behavioural traits). This is the first in a series of three articles describing the distribution of the 41 global DVS worldwide. The remaining two publications will describe those vectors found in (i) Africa, Europe and the Middle East and (ii) in Asia. All geographic distribution maps are being made available in the public domain according to the open access principles of the Malaria Atlas Project.
\end{abstract}

\section{Background}

There is increasing knowledge of the global risk and distribution of Plasmodium falciparum malaria [1] and the intensity of its transmission [2], which reveals the nations of the Americas to have the lowest $P$.

\footnotetext{
* Correspondence: marianne.sinka@zoo.ox.ac.uk; simon.hay@zoo.ox.ac.uk 'Spatial Ecology and Epidemiology Group, Tinbergen Building, Department of Zoology, University of Oxford, South Parks Road, Oxford OX1 3PS, UK Full list of author information is available at the end of the article
}

falciparum malaria endemicity worldwide [2] and hence the lowest $P$. falciparum morbidity $[3,4]$. Work is ongoing to develop the same cartographic suite for $P$. vivax [5]. Data from national health information system reporting support these findings and additionally shows a near cosmopolitan decrease in P. falciparum and $P$. vivax malaria morbidity and mortality across the continents between 2000 and 2007 [6].

\section{() Biomed Central}

(C) 2010 Sinka et al; licensee BioMed Central Ltd. This is an Open Access article distributed under the terms of the Creative Commons Attribution License (http://creativecommons.org/licenses/by/2.0), which permits unrestricted use, distribution, and reproduction in any medium, provided the original work is properly cited. 
Key to these gains, has been the sustained integrated vector control strategy [7-12] championed in the "Regional strategic plan for malaria in the Americas 20062010" [13]. Moreover, these successes and a transformed funding environment [14] have encouraged three of the 21 malaria endemic nations in the Americas (Argentina, El Salvador and Mexico), to scale-up their control ambitions [13] and explicitly target the elimination of malaria within their territories [15]. The global strategic framework for integrated vector management (IVM) calls for "an evidence-based decision-making approach which involves the adaptation of strategies and interventions to local vector ecology, epidemiology and resources that are guided by operational research and subject to routine monitoring and evaluation" [16]. Integrated vector control will therefore remain central to sustaining control impact and become increasingly important for those nations targeting the higher ambition of elimination [17].

Recent attempts to map Anopheles distributions have been made in the Americas [7,18-23]. These include an assessment of mosquito species richness and endemicity in the Neotropical Region (data based on the Mosquito Information Management Project (MIMP) database, incorporating museum specimens dating from 1899 to 1982) [18]; expert opinion ranges for five American DVS, at a continental scale [23]; point data maps detailing the distribution of major malaria vectors in
Venezuela from the early 1900 s to 2005 [21]; the predicted distribution of members of the An. quadrimaculatus Subgroup [19]; the mapped results of a 35-year anopheline survey effort across Panama [20]; and an eco-regional map with details of each region's associated vectors [22]. The evidence-base of occurrence records is not always provided and the mapping techniques used range from those based on expert opinion and simple interpolations to those employing more sophisticated statistical methods. Consequently, the resulting maps are difficult to compare and impossible to synthesize at the continental scale. In addition, species sympatry often obscures the relative contribution to local human malaria transmission. These interactions can, in part, be clarified by an overview of the life history characteristics (bionomics) of vector species pertinent to epidemiology and control.

The current paper focuses on the work carried out on the nine dominant vector species (DVS) identified in the Americas [24] (Table 1). Occurrence data assemblies, expert opinion map compilation and range predictions for each species (or species complex) are presented, alongside a summary of the behaviour and bionomics relevant to local malaria epidemiology and intervention. The DVS of other geographical regions [24] including Africa, Europe and the Middle-East (Sinka et al: The dominant Anopheles vectors of human malaria in Africa, Europe and the Middle East: occurrence data,

Table 1 Defining the dominant Anopheles vector species and species complexes of human malaria in the Americas

\begin{tabular}{|c|c|c|c|c|c|c|c|c|}
\hline $\begin{array}{l}\text { Anopheline species or } \\
\text { species complex }\end{array}$ & $\begin{array}{l}\text { White } \\
\text { [29] }\end{array}$ & $\begin{array}{l}\text { Service } \\
{[27,28]}\end{array}$ & $\begin{array}{l}\text { Kiszewski } \\
{[25]}\end{array}$ & $\begin{array}{l}\text { Mouchet } \\
{[26]}\end{array}$ & Exc. & Inc. & $\begin{array}{l}\text { TAG } \\
\text { final }\end{array}$ & EO source \\
\hline $\begin{array}{l}\text { albimanus Wiedemann, } \\
\text { 1820: An. (Nyssorhynchus) }\end{array}$ & $1,2,3$ & $1,2,3$ & $1,2,3$ & $1,2,3$ & 1 & 1 & 1 & $\begin{array}{l}\text { Rubio-Palis (unpub. obs., 2008), Manguin (unpub. obs., } \\
\text { 2009), Fernandez (unpub. obs., 2009), Updated by TAG } \\
\text { (2009). }\end{array}$ \\
\hline $\begin{array}{l}\text { albitarsis*: An. } \\
\text { (Nyssorhynchus) }\end{array}$ & 3 & 3 & & & & 1 & 1 & $\begin{array}{l}\text { [94], [200], Rubio-Palis (unpub. obs., 2009), Manguin } \\
\text { (unpub. obs., 2009), Updated by TAG (2009). }\end{array}$ \\
\hline $\begin{array}{l}\text { aquasalis Curry, 1932: An. } \\
\text { (Nyssorhynchus) }\end{array}$ & 2,3 & 2,3 & 2,3 & 2,3 & 1 & 1 & 1 & [26], Rubio-Palis (unpub. obs., 2008). \\
\hline $\begin{array}{l}\text { darlingi Root, 1926: An. } \\
\text { (Nyssorhynchus) }\end{array}$ & 2,3 & 2,3 & 2,3 & 2,3 & 1 & 1 & 1 & $\begin{array}{l}\text { [26], Rubio-Palis (unpub. obs., 2008), Updated by TAG } \\
\text { (2009). }\end{array}$ \\
\hline $\begin{array}{l}\text { freeborni Aitken, 1939: An. } \\
\text { (Anopheles) }\end{array}$ & 1 & 1 & 1 & & & 1 & 1 & {$[201]$} \\
\hline $\begin{array}{l}\text { marajoara Galvão \& } \\
\text { Damasceno, 1942: An. } \\
\text { (Nyssorhynchus) }\end{array}$ & & & 2,3 & & & 1 & 1 & $\begin{array}{l}\text { [200], [202], Rubio-Palis (unpub. obs., 2009), Manguin } \\
\text { (unpub. obs., 2009). }\end{array}$ \\
\hline $\begin{array}{l}\text { nuneztovari*: An. } \\
\text { (Nyssorhynchus) }\end{array}$ & 3 & 3 & 3 & 3 & 1 & 1 & 1 & $\begin{array}{l}\text { [26], Rubio-Palis (unpub. obs., 2009), Updated by TAG } \\
\text { (2009). }\end{array}$ \\
\hline $\begin{array}{l}\text { pseudopunctipennis*: An. } \\
\text { (Anopheles) }\end{array}$ & $1,2,3$ & $1,2,3$ & $1,2,3$ & $1,2,3$ & 1 & 1 & 1 & $\begin{array}{l}\text { [26,148], Rubio-Palis (unpub. obs., 2009), Updated by } \\
\text { TAG (2009). }\end{array}$ \\
\hline $\begin{array}{l}\text { quadrimaculatus Say, 1824: } \\
\text { An. (Anopheles) }\end{array}$ & 1 & 1 & 1 & 1 & 1 & 1 & 1 & [201] \\
\hline
\end{tabular}

* denotes that a "species" is now recognized as a species complex. The exclusive (Exc.) column counts those species identified in all four listed reviews. The inclusive (Inc.) column counts those species identified by any of the four reviews and are therefore the candidate dominant vector species (DVS) considered for mapping. The numbers given in each of the review author columns record the Macdonald malaria epidemiology zone [199] in which the species can be found: 1 - North American; 2 - Central American; 3 - South American. The final DVS species listed were defined during two meetings of a Technical Advisory Group (TAG). $\mathrm{EO}=$ Expert opinion. 
distribution maps and bionomic précis, unpublished) and Asia (Sinka et al: The dominant Anopheles vectors of human malaria in the Asia Pacific region: occurrence data, distribution maps and bionomic précis, unpublished) will be considered in future publications.

\section{Methods}

\section{Species selection}

The selection of DVS has been previously described [24]. Briefly, a series of authoritative review articles were consulted [25-29], and those Anopheles vectors identified as main, dominant or principal were assembled to give an inclusive list of 52 species globally. This list was further refined in consultation with a Technical Advisory Group (TAG) of vector experts to give a final list of 41 species and species complexes worldwide, nine of which are found in the Americas (Table 1) [24].

\section{Data assembly - maps}

The Malaria Atlas Project (MAP) parasite rate data library was searched first [30], augmented with a systematic search, conducted in January 2007, for published, peer-reviewed literature detailing primary anopheline vector occurrence data. Online scientific bibliographic databases (PubMed [31] and Web of Science [32]) were searched for post 31 December 1984 articles using "Anopheles" as the keyword search term. In addition, relevant electronic archives were also searched, including AnoBase [33], the Walter Reed Biosystematics Unit (WRBU) Mosquito Catalog [34], the Disease Vectors Database [35], archives of MalariaWorld [36] and Malaria in the News [37], and a review of selected bibliographies [26].

The resulting citation library was then reviewed and refined, retaining all references that met the following criteria for inclusion: (i) the reported study was undertaken after December 1984 (longitudinal surveys that began prior to but continued past this date were included); (ii) the surveys provided location information to a precision of administrative unit level one or higher; (iii) the surveys reported primary data; (iv) the surveys provided species-level information at the studied location; and (v) the surveys reported the presence of at least one DVS. Electronic mail alerts were then set up on various malaria information bulletins (Malaria in the News [37], Malaria World [36] and the Malaria Bulletin [38]) and by using "Anopheles" as a key word to generate article alerts via PubMed [31]. Content notifications for specific high yielding journals including Malaria Journal and Parasite and Vectors (via BioMed Central [39]), Vector-Borne and Zoonotic Diseases [40] and the Indian Journal of Medical Research [41] were also set up. Results from these searches were included until 31 October 2009.
Globally, the literature search resulted in 3857 publications or reports containing potential data to be reviewed. Of these publications, 2276 fulfilled the inclusion criteria, providing data for 147 countries worldwide. A total of 366 sources detailed surveys conducted across 25 countries in the Americas that are further summarized in the results. These data were then augmented with species records from the WRBU MosquitoMap [42].

\section{Expert opinion distribution maps}

Expert opinion (EO) maps were digitised from exhaustive searches of published distribution maps (Table 1). These were then refined by the TAG of Anopheles experts (see acknowledgements) during a meeting held in Oxford (23-25 September 2009). A compressed file containing these EO distributions in ArcGIS format is provided as Additional file 1: Expert opinion distribution maps for the nine DVS of the Americas.

\section{Database}

The data were extracted into Microsoft Excel datasheets using a protocol provided elsewhere [24]. In brief, from each source article, the first author, date of publication, publication type (published article, thesis, unpublished report, etc.) were recorded. Vector- and survey-specific data abstracted included: survey location(s) including country- and administration-level information where given; whether the survey was conducted in an urban or rural location (as defined in the data source); the presence of forest or rice growing areas within the survey area (as defined in the data source); month, year and duration of the survey including information relating to multiple or longitudinal surveys where reported; species data, including sibling information where given; all vector sampling techniques employed in the survey (e.g. human landing indoors and outdoors, animal bait, resting indoors and outdoors); all identification methods (e.g. morphology, polymerase chain reaction (PCR) methods, cross-mating); and any control programs or methods currently in place in the survey location(s) (e.g. insecticide treated bednets (ITNs), insecticide residual spraying (IRS) or larvicide applications). No assumptions were made in the data abstraction, with all reported data accurately reflecting the level of detail given in the data source. For example, where a methodology described a series of monthly surveys conducted over a year, but the results were amalgamated and presented for the whole survey period, these data were abstracted reflecting the whole survey period. Monthly data were only recorded where survey results specifically reported the presence of the vector species in question during that month's survey. 
The data then underwent a three-level checking procedure, with the first level check conducted by a different abstractor to ensure an independent assessment of the assembled data. All aspects of the data were reviewed to ensure the information had been correctly collated and the sites geo-referenced accurately. Detailed notes were included to describe how each line of data had been checked and where changes had been made. The second-level check (by MES) incorporated suggested changes with an emphasis on geo-referencing and the examining of evidence given in support of the choice of coordinates.

Once these first- and second-level checks were completed, the Excel sheets were migrated into a web-based PostgreSQL database [43] with a custom-designed Python interface (based on Django [44]). The Python interface is important because it allows automated production of the occurrence data maps for review, and communication between the database and the PyMC statistical language ultimately used to produce the predicted ranges.

Third-level checks were implemented using the database to identify: (i) inconsistent or non-standard spellings within fields that would affect query summaries; (ii) blanks in mandatory data fields; (iii) any occurrence data that fell in the sea or other major water body (those falling in water bodies less than $1 \times 1 \mathrm{~km}$ from the land were automatically adjusted, any points falling a distance greater than $1 \times 1 \mathrm{~km}$ from the land were manually checked and corrected); (iv) any suspect geo-locations that fell outside the allocated country boundary; (v) all multi-point locations within a point or wide-area area type (e.g. where a survey listed three georeferenced villages, but the data were reported across the whole survey area) in order to identify the point most central to the entire survey area.

Preliminary maps were produced, displaying the EO ranges and the occurrence data. Any points that fell outside the EO outline were checked and accepted as valid, or rejected, based on information provided by the TAG. The final occurrence data and EO maps were collated to show the extent of the geographical distribution of each species and to allow gaps in the data retrieved to be identified [24]. These are shown as thumbnails in the predictive maps in Additional file 2: Predictive species distribution maps for the nine DVS of the Americas.

\section{Boosted Regression Tree modelling}

A wide range of approaches have been developed for empirical modelling of species distributions given data on point observations of occurrence. In this study, the Boosted Regression Tree (BRT) method [45,46] was chosen to generate predictive maps of Anopheles species distribution. This selection was based on a number of factors: first, in a review of 16 species modelling methods, BRT was one of the top performing methods evaluated using the Area Under the receiver operating characteristic Curve (AUC) and correlation statistics [47]; second, the method is flexible in being able to accommodate different types of predictor variables (e.g. continuous or categorical data); third, it is easy to understand and uses reliable, well documented and freely available $\mathrm{R}$ code; and fourth, the resulting maps are simple to interpret and include a ranked list of environmental predictors.

A full description of the BRT method is given in Elith et al. [45]. Briefly, BRTs combine regression or decision trees and "boosting". Regression trees use binary recursive partitioning to iteratively split the data into partitions. Put simply, the model uses the data (in this case presence and pseudo-absence (see below) of a mosquito species) and, in a series of steps, identifies the threshold of each input variable that results in either the presence or the absence of the species. It allows the input of continuous and categorical variables and different scales of measurement amongst the predicting variables.

Boosting is a machine-learning algorithm that increases a model's accuracy $[45,46,48]$. This is applied to the regression trees to help improve their predictive performance. Boosting is based on the principle that in machine learning, a set of "weak" rules can create a single "strong" or accurate rule, and it is easier to create many of these weak rules than it is to find a single accurate rule. For example, it is easy to generalise and state that, as a rule of thumb, a certain anopheline species is never found above a certain altitude, however, accurately specifying the exceptions to this rule across the species' range is considerably more difficult. In the case of BRT, a set of regression trees, each describing the data to a greater or lesser extent, are combined, or boosted, to create a single model with a higher predictive performance than each of its composing parts.

The boosting process within the BRT attempts to minimise a loss function, in this case deviance (two times the $\log$ of the ratio of the likelihood of the data, given the fitted model, and the likelihood of the data, given a perfectly fitting model) that indicates the poor performance of a weak model. Starting with the regression tree within the set that explains the most deviance, additional trees are added that best fit the residuals, or unexplained deviance, of the first tree. The residuals of this new two-tree model are recalculated and the next tree is added to fit these updated residuals, and so on. This stage-wise method could theoretically continue until the model is completely over-fitted, and predictive performance lost. To prevent such over-fitting, shrinkage (reducing the contribution of each tree) is applied to each new tree as it is added which involves the 
optimisation of the number of trees within the final model, the learning rate of the boosting (which is inversely related to the number of trees in the model), and the final tree complexity. This optimisation is achieved using cross-validation, where the model is tested using a sub-sample of withheld data, before the final model is run using all the data, applying the predetermined optimal settings.

\section{Evaluation statistics}

Deviance (see above), Correlation, Discrimination (AUC) and Kappa $(\kappa)$ summary statistics were calculated to accompany each map combining classic accuracy metrics (e.g. Kappa) and those more specific to, and calculated by, the BRT method and recommended for use in Elith et al. [45] (e.g. Deviance). These statistics are used here as a guide to the predictive performance of the maps, but not to compare one map to another, as they were each generated using different datasets.

During cross-validation, BRT repeatedly partitions the data into training and testing subsets and performs cross-validation on each of the different partitions (or folds). It then calculates the mean and standard error for each statistic. The deviance metric is the prime measure of accuracy for the BRT method, and indicates the proportion of deviance still unexplained by the model. The lower the deviance value (minimum value $=0$ ), the better the model is at predicting the hold-out sets. Correlation simply correlates the predicted probability of presence against the occurrence data, giving a value between -1 and 1 . The area under the curve (AUC) of the receiver-operator characteristic is also presented as an accuracy metric. The AUC provides values ranging between 0 and 1 , where 1 is a perfect prediction, and is an index of the area under the curve of a plot of sensitivity (the proportion of the testing set that is correctly classified as a presence) against specificity (the proportion that is correctly classified as an absence) as the classification threshold is increased [49]. Kappa is an index of the proportion of agreement of predicted versus observed positive and negative samples, calculated from an error matrix that cross references the number of observed and the number of predicted pixels categorised as presence or absence [49-51]. It can provide values ranging from -1 to 1 , where values less than zero indicate that the model is worse than random, zero shows that the model is no better than random, and greater than zero indicates that the model performs better than random.

\section{Environmental and climatic variables}

Each of the environmental or climatic data grids described below have undergone a number of processing steps prior to being used in the BRT. Each grid was post-processed to ensure that the pattern of land and sea pixels corresponded exactly across all grids, in order to provide a suite of spatially identical grids. This required trimming or gap-filling via nearest-neighbour interpolation to reconcile minor misalignments in coastline definitions or, for example, small data gaps due to cloud error.

Covariates were chosen based on factors known to influence anopheline ecology and therefore include elevation [52], climatology surfaces interpolated from networks of meteorological stations [53], and remotely sensed data from Earth observation satellites in their raw form and categorised into global land cover maps $[54,55]$. Where remotely sensed imagery was available as multi-temporal data, temporal Fourier analysis (TFA) was used to ordinate the data by decomposing the temporal signal into an additive series of harmonics of different seasonal frequencies [56]. The TFA algorithm [56] generated seven products for each temporal variable: the overall mean, maximum and minimum of the data cycles; the amplitude (maximum variation of the cycle around the mean) and the phase (the timing of the cycle) of the annual and bi-annual cycles. The grids are all described in detail below.

\section{Elevation}

The Shuttle Radar Topography Mission (SRTM), consisting of a synthetic aperture radar on board the Space Shuttle Endeavour, flew an 11 day mission to create a near-global high spatial resolution digital elevation model (DEM) [52,57]. The DEM grids used here are processed by applying land/sea masks and the data are projected into the MODIS Land (MODLAND) tile system. The MODerate Resolution Imaging Spectroradiometer (MODIS) is a NASA satellite sensor which provides $1 \times 1 \mathrm{~km}$ spatial resolution satellite imagery globally [58].

\section{Worldclim database}

The Worldclim database consists of a freely available set of global climate data at a $1 \times 1 \mathrm{~km}$ spatial resolution compiled using weather data collected from world-wide weather stations [53]. The data spans from 1950-2000 and describes monthly precipitation and mean, minimum and maximum temperatures. From these data, interpolated climate surfaces have been produced using ANUSPLIN-SPLINA software [59].

\section{Advanced Very High Resolution Radiometer}

The Advanced Very High Resolution Radiometer (AVHRR) $8 \times 8 \mathrm{~km}$ products are available over a 20 year time series, and a limited series of $1 \times 1 \mathrm{~km}$ resolution data are only available for April to December 1992; January to September 1993; February to December 1995 
and January to April 1996. Both data series were downloaded (Goddard Space Flight Center's Distributed Active Archive Center on the Global Land Biosphere Data and Information Web Site [54]) and processed for use here [60].

The AVHRR grids used include the normalized difference vegetation index (NDVI), land surface temperature (LST) and middle infrared radiation (MIR). The NDVI numerically indicates the level of green, photosynthesizing, and therefore active, vegetation derived from the spectral reflectance of AVHRR channels 1 and 2 (visible red and near infrared wavelength, respectively) $[61,62]$. The LST index uses thermal infrared radiometry to measure land temperature, corrected for atmospheric influences, such as water vapour, aerosols, carbon dioxide or ozone $[61,63]$. Finally, the MIR data is applied to discriminating land cover. It is able to penetrate more fully than shorter wavelengths through aerosol particles, including atmospheric water, and it is considered better able to distinguish between vegetation, soil, rock and water $[61,64]$.

\section{Globcover project}

The Globcover project [55] provides satellite-derived land cover maps from the MERIS spectrometer on board ENVISAT. The data produced are at a $300 \times 300$ $m$ resolution and the satellite imagery goes through a number of pre-processing and classification steps prior to map production, which include cloud screening and shadow detection, water reclassification and atmosphere (including aerosol) correction. The final map products include global land cover mosaics for the period from December 2004 to June 2006, providing 22 land cover classes; and regional mosaics, which detail up to 51 land cover classes. Due to the limited areas covered by the regional mosaics, the global mosaics are used here. They include land-cover classes particularly relevant to mosquito habitats, for example, post-flooding or irrigated croplands, rain-fed croplands, urban areas and numerous categories for forests, including those regularly flooded with fresh or saline/brackish water. The 22 categories were available to the BRT individually and also grouped into three land cover types: flooded areas, forested areas and dry areas. Finally, to produce grids of an equivalent resolution to the other environmental and climatic variables applied in the vector mapping $(5 \times 5$ $\mathrm{km}$ ), the $300 \mathrm{~m}$ mosaics were re-sampled using a majority filter where the most common class in each pixel subset was identified and used to redefine the new, larger pixel.

The covariate sources detailed above provide a range of spatial, high resolution environmental or climatic open source data freely available for species mapping. All grids were available to the BRT mapping process allowing the model to identify which variable, or suite of variables most accurately described the species distribution.

\section{Mapping protocol}

Numerous model iterations were run with varying combinations of buffer size, number of pseudo-absences and source data (hybrid data: occurrence data plus pseudopresences randomly assigned from within the EO species range; EO data: pseudo-presences randomly assigned from within the EO range; and occurrence data only). As each of these categories required the use of different data inputs to the BRT, statistical comparison using the evaluation metrics was not possible. Therefore the "optimal" settings chosen are subjective and based on visual examination and comparison of the various maps guided by, but not relying on, the evaluation statistics.

The lack of true absences in the occurrence data is a common problem encountered with species mapping and can be addressed by the production of pseudoabsences. These can be created in a number of ways [65] including creating background samples within the region of study $[47,66]$ to represent the set of conditions available to the species, though not necessarily areas where the species is genuinely absent. Alternatively, and to minimise the risk of including unidentified presence data, pseudo-absences can be assigned within a buffer zone surrounding the region of study $[49,67]$. This second method is particularly useful where the extent of the species range to be mapped is known or can be reasonably estimated, for example with expert opinion maps. The buffer in such cases represents an area that is not geographically implausible for the species to reach, but maybe outside the limit of the environment that the species can tolerate. The creation of pseudo-absences in the buffer zone was used here.

The size of the buffer zone can have implications for the accuracy and viability of the final mapping outputs [67]. A buffer that is too large, allowing pseudo-absences to be generated far from the presence data, may result in a model that is defining regional or coarse geographical differences rather than the fine-scale variables that influence the presence or absence of a species. Conversely, a buffer that is too small, which relies on pseudoabsences generated too close to the presence data, risks incorporating unknown areas of presence, severely limiting the ability of the model to distinguish presence or absence. Both scenarios result in poor model performance and variable selection, and hence poor predictive ability [67]. To identify the optimal buffer size, maps were run for all species with buffer sizes of $100 \mathrm{~km}, 500$ $\mathrm{km}, 1000 \mathrm{~km}$ and $1500 \mathrm{~km}$, using 1000 pseudo-absences 
(see below). These maps were then visually and statistically compared.

The number of pseudo-absences generated in the mapping process can also influence the final mapping product. As a rule of thumb, a proportionally greater number of pseudo-absences to presence data should be used to overcome the possibility that within the randomly selected absences, some may fall on true, yet currently unidentified, presence points [49]. To establish the optimal number of pseudo-absences to use in the mapping, maps for all nine DVS were created and compared using 1:1, 2:1, 5:1 and 10:1 ratios of pseudoabsences to presence data, and additionally using a constant number of 500 and 1000 pseudo-absences for all species. These maps were then visually and statistically compared.

The predictive value of the expert opinion maps were tested using two methods: (i) for each species, 500 pseudo-presences, randomly distributed within the expert opinion species range boundary, were created and run through the BRT mapping alongside 500 pseudo-absences from within the buffer area. Using these pseudo-data, maps were created to identify whether the boundaries given in the expert opinion maps would be recreated by the BRT, such as, for example, the dry area indicated in the north-east of Brazil, identified as too dry for the forest/riverine species $A n$. darlingi to survive. (ii) For each species, maps were created using the real occurrence data and, firstly, 500 pseudo-absences randomly selected from the buffer zone and of the same weighting as the true data, and, secondly 500 pseudo-presence points randomly assigned within the expert opinion range but with a weighting half that of the true data plus 500 pseudo-absences created as before. Maps created using both methods were again compared.

All mapping trails were conducted using environmen$\mathrm{tal} /$ climatic variable grids of $5 \times 5 \mathrm{~km}$ resolution, allowing the production of high quality maps within feasible periods of time.

\section{Data assembly - bionomics}

The need to understand the behaviour and life history characteristics or "bionomics" of the DVS is clear. The impact of interventions, such as ITNs, and control, such as IRS, is closely related to the behavioural characteristics of the local DVS. In addition, such information can help rank the relative importance of a species in an area with regards to transmission and be of major importance to the parameterisation of mathematical models. No recent comprehensive summary of these information exist.

Bionomics information was summarised for each of the nine American DVS (See Additional file 3:
Bionomics protocol, for full methodology). A sub-library for each species was created by searching the citations listed within the MAP library by species name. Where a library contained 30 or more citations, it was filtered using the following terms: "behaviour", "behavior", "larva", "biting", "resting" and "habitat", in order to refine the bibliography to include only studies detailing behavioural/bionomic information (Table 2).

Each of the articles listed in each sub-library was read, and all relevant behavioural information extracted and summarised (Additional file 3: Bionomics protocol). The TAG were consulted and also provided a summary of their knowledge of the bionomics of each DVS which was combined with the relevant literature summary to detail general larval site characteristics (Table 3), known larval habitat types (Tables 4-5), and adult biting and resting behaviour (Table 6).

The bionomics review does not include any detailed information relating to insecticide resistance. This was purposely omitted as this highly dynamic and important aspect of the vectors is being addressed in detail by other groups including the Innovative Vector Control Consortium (IVCC) [68] and groups at the Liverpool School of Tropical Medicine.

\section{Results}

\section{Data per species}

Data were collected in 25 countries in the Americas (Table 7) from which 5408/5942 occurrence points and 2889/3257 sites have been geo-referenced (summary numbers generated in April 2010). "Occurrence" includes all temporal data, where given, and "sites" refers to unique locations. For example, if a study reports the presence of a species every month for a year, this gives 12 incidences of occurrence, but only one georeferenced site. Data were categorised into the area types that define the size of the area sampled [30]. There were 1509 point locations $\left(\leq 10 \mathrm{~km}^{2}\right), 88$ wide areas $\left(10-25 \mathrm{~km}^{2}\right), 42$ small polygons $\left(25-100 \mathrm{~km}^{2}\right)$ and 369 large polygons $\left(>100 \mathrm{~km}^{2}\right)$ (Table 7$)$ in the Americas.

Adult collections were reported from 1203 locations, larval collections from 633 and a combination of larval and adult collections from 377 sites. A total of 595 sites reported the presence of more than one DVS, with a maximum of five co-existing species found at San Pedro de Uraba, Colombia. The most popular sampling method of all the reported studies was outdoor landing catches using human bait (MBO). This method was used in 110 studies and at 411 sites. Across the Americas, human landing (indoors and outdoors) sampling techniques were used in $39.3 \%$ of studies and at $24.8 \%$ of sites compared to the $2.7 \%$ of studies and only $1.9 \%$ of sites that used animal bait collections. Similar 
Table 2 Citation search results for the bionomics survey taken from the MAP database

\begin{tabular}{lllll}
\hline Species & Citations (unfiltered) & Citations (filtered) & Citations with data & References. \\
\hline An. albimanus & 111 & 68 & 24 & {$[73,76-91,185,203-209]$} \\
\hline An. albitarsis & 73 & 42 & 15 & {$[74,93,95,96,98-105,140,210,211]$} \\
\hline An. aquasalis & 30 & 20 & 10 & {$[93,100,106-109,111,112,212-214]$} \\
\hline An. darlingi & 138 & 78 & 36 & {$[73-75,90,95,104,113-123,131,141,185,208,210,215-229]$} \\
\hline An. freeborni & 19 & - & 3 & {$[127,128,230]$} \\
\hline An. marajoara & - & - & 6 & {$[113,116,117,129,131,132,220]$} \\
\hline An. nuneztovari & 53 & 28 & 10 & {$[74,80,95,99,102,116,131,139-141,145]$} \\
\hline An. pseudopunctipennis & 46 & 32 & 20 & {$[76,93,109,139,148,149,151-159,208,212,231-234]$} \\
\hline An. quadrimaculatus & 66 & 36 & 16 & {$[69,160-171,235-237]$} \\
\hline
\end{tabular}

Filter terms were: behaviour, behavior, larva, biting, resting and habitat. Anopheles albitarsis and An. marajoara libraries were combined prior to data extractions to try and ensure no information was omitted (An. marajoara is a sibling of the An. albitarsis complex). Articles/data specific to An. marajoara were listed in the datasheet separately and those listed under "An. albitarsis" are for all other members of the complex, excluding An. marajoara

numbers of studies searched for resting mosquitoes inside and outside houses $(5.1 \%$ resting indoors and $5.2 \%$ resting outdoors). However, a greater number of sites were searched for outdoor resting mosquitoes in these studies (16.4\% compared to $5.1 \%$ resting indoors), although this is a consequence of two large studies that searched for An. quadrimaculatus in the USA: Reinert et al. [69], 235 sites searched; Seawright et al. [70], 148 sites searched. Light trapping or carbon dioxide bait was only used at $4.4 \%$ of sites. Morphological identification methods were used at 1102 sites, with PCR techniques applied to identify samples from 178 sites. Longitudinal data were recorded where given, and within these data, 139 studies reported DVS occurrence sampled for over

Table 3 Larval site characteristics

\begin{tabular}{|c|c|c|c|c|c|c|c|c|c|c|c|}
\hline \multirow[t]{2}{*}{ Species } & \multirow[t]{2}{*}{ Source } & \multicolumn{2}{|c|}{ Light intensity } & \multicolumn{2}{|c|}{ Salinity } & \multicolumn{2}{|c|}{ Turbidity } & \multicolumn{2}{|c|}{ Movement } & \multicolumn{2}{|c|}{ Vegetation } \\
\hline & & $\begin{array}{l}\text { Helio- } \\
\text { philic }\end{array}$ & $\begin{array}{l}\text { Helio- } \\
\text { phobic }\end{array}$ & $\begin{array}{c}\text { High } \\
\text { (brackish) }\end{array}$ & $\begin{array}{c}\text { Low } \\
\text { (fresh) }\end{array}$ & Clear & Polluted & $\begin{array}{c}\text { Still or } \\
\text { stagnant }\end{array}$ & Flowing & $\begin{array}{l}\text { Higher plants, } \\
\text { algae etc. }\end{array}$ & $\begin{array}{l}\text { No } \\
\text { Veg }\end{array}$ \\
\hline An. albimanus & Summary & 3 & & 4 & 4 & 1 & & 3 & 3 & 12 & \\
\hline An. albimanus & TAG & $\bullet$ & & ० & $\bullet$ & $\bullet$ & & $\bullet$ & ○ & $\bullet$ & \\
\hline An. albitarsis & Summary & & & & 1 & 2 & 2 & 1 & & 2 & \\
\hline An. albitarsis & TAG & $\bullet$ & & & $\bullet$ & $\bullet$ & ० & $\bullet$ & & $\bullet$ & \\
\hline An. aquasalis & Summary & 2 & & 3 & 2 & 1 & 1 & 2 & 1 & 3 & \\
\hline An. aquasalis & TAG & $\bullet$ & & $\bullet$ & $\bullet$ & $\bullet$ & & $\bullet$ & & $\bullet$ & \\
\hline An. darlingi & Summary & 1 & 6 & 1 & 1 & 2 & 1 & 4 & 4 & 8 & \\
\hline An. darlingi & TAG & $\circ$ & $\bullet$ & & $\bullet$ & $\bullet$ & & $\bullet$ & $\bullet$ & $\bullet$ & \\
\hline An. freeborni & Summary & & & & & & & & & & \\
\hline An. freeborni & TAG & $\bullet$ & & & $\bullet$ & $\bullet$ & & $\bullet$ & & $\bullet$ & \\
\hline An. marajoara & Summary & 2 & & & & 1 & 1 & 1 & & & \\
\hline An. marajoara & TAG & $\bullet$ & O & & $\bullet$ & $\bullet$ & & $\bullet$ & $\bullet$ & $\bullet$ & \\
\hline An. nuneztovari & Summary & 3 & 3 & & & 2 & 2 & & & 1 & \\
\hline An. nuneztovari & TAG & $\bullet$ & $\bullet$ & & $\bullet$ & $\bullet$ & & $\bullet$ & $\bullet$ & $\bullet$ & \\
\hline $\begin{array}{l}\text { An. } \\
\text { pseudopunctipennis }\end{array}$ & Summary & 2 & & 3 & 2 & 2 & 1 & 2 & 1 & 10 & \\
\hline $\begin{array}{l}\text { An. } \\
\text { pseudopunctipennis }\end{array}$ & TAG & $\bullet$ & & ० & $\bullet$ & $\bullet$ & ० & $\bullet$ & ० & $\bullet$ & \\
\hline $\begin{array}{l}\text { An. } \\
\text { quadrimaculatus }\end{array}$ & Summary & & & & 2 & 1 & 1 & 2 & & 8 & 1 \\
\hline $\begin{array}{l}\text { An. } \\
\text { quadrimaculatus }\end{array}$ & $\mathrm{TAG}$ & $\bullet$ & & & $\bullet$ & $\bullet$ & & $\bullet$ & & $\bullet$ & \\
\hline
\end{tabular}

TAG: Rubio-Palis \& Manguin (unpub. obs., 2009, 2010), $\bullet=$ typical, $\circ=$ examples exist. Numbers indicate the number of studies that found larvae under each listed circumstance. Anopheles albitarsis refers to the An. albitarsis complex, which includes An. albitarsis, An. albitarsis sp. B, sp. E and An. deaneorum. Anopheles marajoara is listed separately. 
Table 4 Large larval sites

\begin{tabular}{|c|c|c|c|c|c|c|c|c|c|c|c|c|}
\hline \multirow[t]{2}{*}{ Species } & \multirow[t]{2}{*}{ Source } & \multicolumn{6}{|c|}{ Large natural water collections } & \multicolumn{5}{|c|}{ Large man-made water collections } \\
\hline & & Lagoons & Lakes & Marshes & Bogs & $\begin{array}{c}\text { Slow flowing } \\
\text { rivers }\end{array}$ & Other & $\begin{array}{l}\text { Borrow } \\
\text { pits }\end{array}$ & $\begin{array}{l}\text { Rice } \\
\text { fields }\end{array}$ & $\begin{array}{l}\text { Fish } \\
\text { ponds }\end{array}$ & $\begin{array}{l}\text { Irrigation } \\
\text { channels } \\
\end{array}$ & Other \\
\hline An. albimanus & Summary & 5 & 1 & 5 & 1 & 1 & 7 & & 2 & 1 & 3 & 1 \\
\hline An. albimanus & TAG & $\bullet$ & $\circ$ & $\bullet$ & $\circ$ & & & $\bullet$ & & & & \\
\hline An. albitarsis & Summary & 2 & 1 & & & & 1 & & 1 & & & \\
\hline An. albitarsis & TAG & $\bullet$ & $\bullet$ & $\bullet$ & & & & & $\bullet$ & & & \\
\hline An. aquasalis & Summary & 1 & & 2 & & & 4 & & & & 1 & \\
\hline An. aquasalis & TAG & $\bullet$ & & $\bullet$ & $\bullet$ & & & & $\bullet$ & & $\bullet$ & \\
\hline An. darlingi & Summary & 2 & 2 & & & 3 & 1 & & 1 & & & 2 \\
\hline An. darlingi & TAG & $\bullet$ & & & $\bullet$ & $\bullet$ & & $\bullet$ & & & $\bullet$ & $\circ$ \\
\hline An. freeborni & Summary & 1 & & & & & & & 5 & & & \\
\hline An. freeborni & TAG & & & & & & & & $\bullet$ & & & \\
\hline An. marajoara & Summary & & & & & & 1 & & & 1 & & 1 \\
\hline An. marajoara & TAG & $\bullet$ & $\bullet$ & & $\bullet$ & & & $\bullet$ & $\bullet$ & & $\bullet$ & $\circ$ \\
\hline An. nuneztovari & Summary & 1 & 1 & & & 1 & & & & 2 & & 1 \\
\hline An. nuneztovari & TAG & $\bullet$ & $\bullet$ & & $\bullet$ & $\bullet$ & & $\bullet$ & & $\bullet$ & & $\circ$ \\
\hline $\begin{array}{l}\text { An. } \\
\text { pseudopunctipennis }\end{array}$ & Summary & 3 & & 1 & & 2 & 1 & 1 & & 1 & 1 & 1 \\
\hline $\begin{array}{l}\text { An. } \\
\text { pseudopunctipennis }\end{array}$ & TAG & $\circ$ & & & & $\circ$ & & & & & & \\
\hline $\begin{array}{l}\text { An. } \\
\text { quadrimaculatus }\end{array}$ & Summary & & 4 & 3 & & & 2 & 1 & 9 & & & 2 \\
\hline $\begin{array}{l}\text { An. } \\
\text { quadrimaculatus }\end{array}$ & TAG & $\bullet$ & $\bullet$ & $\bullet$ & & & & & $\bullet$ & & $\bullet$ & \\
\hline
\end{tabular}

TAG: Rubio-Palis \& Manguin (unpub. obs., 2009, 2010), • = typical, $\circ=$ examples exist. Numbers indicate the number of studies that found larvae under each listed circumstance. Anopheles albitarsis refers to the An. albitarsis complex, which includes An. albitarsis, An. albitarsis sp. B, sp. E and An. deaneorum. Anopheles marajoara is listed separately.

a year, relating to 431 sites. A single sampling period was reported from 85 studies, relating to 1235 pointlocation sites, whilst 139 studies reported multiple (two or more) sampling periods from 975 point-locations.

Anopheles darlingi was reported from the greatest number of geo-positioned point locations (488) and had the greatest number of recorded temporal occurrences (859) (Table 8). In comparison, An. freeborni was only reported from 31 site point locations, with only 35 overall occurrence records. The greatest number (377) of point-location sites was recorded from the United States. Guyana had the lowest reported data overall, with only a single point-location reported at the goldmining town of Mahdia in the Amazon interior, where both $A n$. aquasalis and An. darlingi were recorded along with a number of other secondary vector species.

\section{Mapping trials}

All results for each mapping trial are given in Additional file 4: Summary tables showing evaluation statistics for all mapping trials and final BRT environmental and climatic variable selections for the final, optimal predictive maps. Optimal mapping categories were evaluated visually and using the deviance and AUC statistics, with the caveat that these could only be used as a guide rather than a true indication of predictive performance, as different data sets were used for each map produced.

The optimal buffer size within which pseudo-absences were randomly selected was determined to be $1000 \mathrm{~km}$, with five out of nine species maps judged to perform better at this buffer size than those produced using the alternative sizes. A ratio of $5: 1$ pseudo-absences to presence data was applied, taking into account 250 (500 half weighted) pseudo-presences. This level of pseudoabsence input provided enough data to counteract any potential interference that may have been caused by the random inclusion of unidentified presences from within the absence data.

The EO mapping test indicated that where random pseudo-presences are created within the EO range, and no real occurrence data were included, the model would predict a high probability of presence within the whole expert opinion range and calculate a high deviance value for all species, indicating overall, a poor predictive performance. Where the hybrid method was used that incorporated both real occurrence data plus 500 
Table 5 Small larval sites

\begin{tabular}{|c|c|c|c|c|c|c|c|c|c|c|c|c|c|c|c|}
\hline \multirow[t]{2}{*}{ Species } & \multirow[t]{2}{*}{ Source } & \multicolumn{6}{|c|}{ Small natural water collections } & \multicolumn{7}{|c|}{ Small man-made water collections } & \multirow{2}{*}{$\begin{array}{c}\text { Artificial sites } \\
\text { Empty cans, } \\
\text { shells etc. }\end{array}$} \\
\hline & & $\begin{array}{l}\text { Small } \\
\text { streams }\end{array}$ & $\begin{array}{l}\text { Seepage } \\
\text { springs }\end{array}$ & Pools & Wells & $\begin{array}{l}\text { Dips in the } \\
\text { ground }\end{array}$ & Other & $\begin{array}{l}\text { Overflow } \\
\text { water }\end{array}$ & $\begin{array}{l}\text { Irrigation } \\
\text { ditches }\end{array}$ & $\begin{array}{l}\text { Borrow } \\
\text { pits }\end{array}$ & $\begin{array}{l}\text { Wheel } \\
\text { ruts }\end{array}$ & $\begin{array}{l}\text { Hoof } \\
\text { prints }\end{array}$ & $\begin{array}{l}\text { Puddles near } \\
\text { rice fields }\end{array}$ & Other & \\
\hline An. albimanus & Summary & 3 & & 1 & & 1 & 2 & & & & & & & 4 & 3 \\
\hline An. albimanus & TAG & $\bullet$ & & $\bullet$ & $\bullet$ & & & & & $\bullet$ & $\bullet$ & $\bullet$ & & & \\
\hline An. albitarsis & Summary & & & 1 & & & & & & & & & & 3 & 1 \\
\hline An. albitarsis & TAG & $\bullet$ & & $\bullet$ & & $\bullet$ & & & & $\bullet$ & & & & & \\
\hline An. aquasalis & Summary & & & 2 & & & 1 & & & & & & & 1 & 1 \\
\hline An. aquasalis & TAG & $\bullet$ & & $\bullet$ & $\bullet$ & & & & $\bullet$ & & & & $\bullet$ & & \\
\hline An. darlingi & Summary & 3 & & 5 & 1 & & 1 & & & & & & & 1 & \\
\hline An. darlingi & TAG & $\bullet$ & & $\bullet$ & & & & $\bullet$ & & $\bullet$ & & $\bullet$ & & & \\
\hline An. freeborni & Summary & & & & & & 1 & & & & & & & & \\
\hline An. freeborni & TAG & $\bullet$ & & $\bullet$ & & & & & & $\bullet$ & & & & & \\
\hline An. marajoara & Summary & 1 & 1 & & & & & & & & & & & 1 & \\
\hline An. marajoara & TAG & $\bullet$ & & $\bullet$ & & $\bullet$ & & $\bullet$ & $\bullet$ & $\bullet$ & & & $\bullet$ & & \\
\hline An. nuneztovari & Summary & 2 & & 2 & & 1 & & & & & & & 3 & & 1 \\
\hline An. nuneztovari & TAG & $\bullet$ & & $\bullet$ & & $\bullet$ & & & & $\bullet$ & & & & & \\
\hline $\begin{array}{l}\text { An. } \\
\text { pseudopunctipennis }\end{array}$ & Summary & 2 & 2 & 9 & & 1 & & & 1 & & & & & & \\
\hline $\begin{array}{l}\text { An. } \\
\text { pseudopunctipennis }\end{array}$ & TAG & $\bullet$ & $\circ$ & $\bullet$ & & & & & $\circ$ & & & & & & \\
\hline $\begin{array}{l}\text { An. } \\
\text { quadrimaculatus }\end{array}$ & Summary & 1 & & 2 & & & 2 & & & 1 & & & & 1 & 2 \\
\hline$A n$ & TAG & $\bullet$ & & & & & & & $\bullet$ & $\bullet$ & & & & & \\
\hline
\end{tabular}

TAG: Rubio-Palis \& Manguin (unpub. obs., 2009, 2010), $\bullet=$ typical, $\circ=$ examples exist. Numbers indicate the number of studies that found larvae under each listed circumstance. Anopheles albitarsis refers to the An. albitarsis complex, which includes An. albitarsis, An. albitarsis sp. B, sp. E and An. deaneorum. Anopheles marajoara is listed separately. 
Table 6 Adult feeding and resting behaviour

\begin{tabular}{|c|c|c|c|c|c|c|c|c|c|c|c|c|c|}
\hline \multirow[t]{2}{*}{ Species } & \multirow[t]{2}{*}{ Source } & \multicolumn{2}{|c|}{ Feeding habit } & \multicolumn{2}{|c|}{ Biting habit } & \multicolumn{4}{|c|}{ Biting time } & \multicolumn{2}{|c|}{$\begin{array}{l}\text { Pre-feeding } \\
\text { resting habit }\end{array}$} & \multicolumn{2}{|c|}{$\begin{array}{l}\text { Post-feeding } \\
\text { resting habit }\end{array}$} \\
\hline & & $\begin{array}{l}\text { Anthro- } \\
\text { pophilic }\end{array}$ & $\begin{array}{l}\text { Zoo- } \\
\text { philic }\end{array}$ & $\begin{array}{l}\text { Exo- } \\
\text { phagic }\end{array}$ & $\begin{array}{l}\text { Endo- } \\
\text { phagic }\end{array}$ & Day & Dusk & Night & Dawn & $\begin{array}{l}\text { Exo- } \\
\text { philic }\end{array}$ & $\begin{array}{l}\text { Endo- } \\
\text { philic }\end{array}$ & $\begin{array}{l}\text { Exo- } \\
\text { philic }\end{array}$ & $\begin{array}{l}\text { Endo- } \\
\text { philic }\end{array}$ \\
\hline An. albimanus & Summary & 2 & 2 & 9 & 2 & & 7 & 9 & 0 & & & 1 & 3 \\
\hline An. albimanus & TAG & $\bullet$ & $\bullet$ & $\bullet$ & $\bullet$ & & $\bullet$ & $\bullet$ & & $\bullet$ & & $\bullet$ & \\
\hline An. albitarsis & Summary & 2 & 2 & 4 & 3 & & 7 & 3 & & & & 2 & \\
\hline An. albitarsis & TAG & $\bullet$ & $\bullet$ & $\bullet$ & $\bullet$ & & $\bullet$ & $\bullet$ & & $\bullet$ & & $\bullet$ & $\circ$ \\
\hline An. aquasalis & Summary & 1 & 1 & 2 & 2 & 1 & 2 & 1 & & 1 & & 1 & \\
\hline An. aquasalis & TAG & $\bullet$ & $\bullet$ & $\bullet$ & $\bullet$ & & $\bullet$ & $\bullet$ & & $\bullet$ & & $\bullet$ & \\
\hline An. darlingi & Summary & 12 & & 9 & 6 & & 15 & 23 & 3 & 1 & & 2 & \\
\hline An. darlingi & TAG & $\bullet$ & $\circ$ & $\bullet$ & $\bullet$ & & $\bullet$ & $\bullet$ & $\bullet$ & $\bullet$ & & $\bullet$ & \\
\hline An. freeborni & Summary & 1 & 1 & & & & & & & & & & \\
\hline An. freeborni & TAG & $\bullet$ & $\bullet$ & $\bullet$ & $\bullet$ & & $\bullet$ & $\bullet$ & $\bullet$ & $\bullet$ & & $\bullet$ & \\
\hline An. marajoara & Summary & 2 & 2 & 3 & & & 4 & 1 & & 1 & & 2 & \\
\hline An. marajoara & TAG & $\bullet$ & $\bullet$ & $\bullet$ & $\bullet$ & & $\bullet$ & $\bullet$ & & $\bullet$ & & $\bullet$ & \\
\hline An. nuneztovari & Summary & 2 & 4 & 5 & 1 & & 3 & 1 & & 1 & & 2 & \\
\hline An. nuneztovari & TAG & $\bullet$ & $\bullet$ & $\bullet$ & $\bullet$ & & $\bullet$ & $\bullet$ & $\bullet$ & $\bullet$ & & $\bullet$ & \\
\hline $\begin{array}{l}\text { An. } \\
\text { pseudopunctipennis }\end{array}$ & Summary & 3 & 2 & 3 & & & & 1 & & & 1 & 1 & 2 \\
\hline $\begin{array}{l}\text { An. } \\
\text { pseudopunctipennis }\end{array}$ & TAG & $\bullet$ & $\bullet$ & $\bullet$ & $\bullet$ & & & $\bullet$ & & $\bullet$ & & $\bullet$ & $\bullet$ \\
\hline $\begin{array}{l}\text { An. } \\
\text { quadrimaculatus }\end{array}$ & Summary & & 3 & 2 & & & 1 & & 1 & 2 & & 2 & \\
\hline $\begin{array}{l}\text { An. } \\
\text { quadrimaculatus }\end{array}$ & TAG & $\bullet$ & $\bullet$ & $\bullet$ & & $\circ$ & $\bullet$ & $\bullet$ & $\bullet$ & $\bullet$ & & $\bullet$ & \\
\hline
\end{tabular}

TAG: Rubio-Palis \& Manguin (unpub. obs., 2009, 2010), $\bullet=$ typical, $\circ=$ examples exist. Numbers indicate the number of studies that found adults under each listed circumstance. Anopheles albitarsis refers to the An. albitarsis complex, which includes An. albitarsis, An. albitarsis sp. B, sp. E and An. deaneorum. Anopheles marajoara is listed separately.

half-weighted pseudo-presence points randomly assigned within the EO range, the mapping performance was greatly improved. Maps created using only the real presence data, produced a low deviance value, but visually, predictive performance was judged to be poor, possibly due to a paucity of data for some species. As with all species mapping, occurrence data will be biased to areas that are accessible for sampling, or as succinctly stated by Coetzee [71], reflect the distribution of entomologists rather than mosquito species. As such, all occurrencebased mapping and mapping evaluations must be treated with some level of caution, especially where the data is sparse. It was thus considered that the hybrid maps performed better overall and are presented here.

\section{Predictive maps}

The BRT species maps for all nine American species are given in Additional file 2: Predictive species distribution maps for the nine DVS of the Americas, and are summarised in Table 9. Spatial constraints prevent all the species being discussed in full here, however Anopheles darlingi, considered one of the most important malaria vectors in the Neotropical Region [23], is reviewed below.

Anopheles darlingi is a lowland, riverine, forest dwelling species (see below and Table 4), unable to survive in dry climates, including for example, north-eastern Brazil (Rio Grande de Norte, Paraiba etc.) as is approximated in the EO map (Figure 1, inset). Figure 1 shows the predicted species distribution using hybrid data (318 occurrence data plus 500 pseudo-presences weighted at half that of the occurrence data and randomly selected from within the EO range) and a 5:1 ratio of pseudo-absence to presence data, taking into account the pseudopresence points. Also indicated are the environmental variables identified by the BRT as being the most influential amongst those applied to the model in describing the species distribution.

Maximum precipitation is the variable identified as having the highest relative influence $(36.31 \%)$ on the presence of $A n$. darlingi compared to $9.04 \%$ for the second-rated variable (maximum LST) (Figure 1; Table 9). Precipitation is selected twice more within the top five (third: mean; and fifth: phase of the bi-annual cycle) and 
Table 7 Number of sites recording species presence per country by area type (points $\left(\leq 10 \mathrm{~km}^{2}\right)$, wide areas (10$\left.25 \mathrm{~km}^{2}\right)$, small $\left(25-100 \mathrm{~km}^{2}\right)$ and large $\left(>100 \mathrm{~km}^{2}\right)$ polygons)

\begin{tabular}{|c|c|c|c|c|}
\hline Country & Point & $\begin{array}{l}\text { Wide- } \\
\text { area }\end{array}$ & $\begin{array}{l}\text { Small } \\
\text { polygon }\end{array}$ & $\begin{array}{l}\text { Large } \\
\text { polygon }\end{array}$ \\
\hline Argentina & 19 & 2 & - & - \\
\hline Belize & 228 & 2 & - & 13 \\
\hline Bolivia & 17 & - & 1 & 2 \\
\hline Brazil & 304 & 16 & 17 & 136 \\
\hline Colombia & 67 & 4 & 6 & 9 \\
\hline Costa Rica & 40 & - & - & 1 \\
\hline Cuba & 5 & 1 & - & 2 \\
\hline $\begin{array}{l}\text { Dominican } \\
\text { Republic }\end{array}$ & 5 & 1 & 2 & - \\
\hline Ecuador & 19 & - & - & - \\
\hline El Salvador & 3 & - & - & - \\
\hline French Guiana & 7 & - & 1 & - \\
\hline Grenada & 17 & 1 & - & - \\
\hline Guatemala & 51 & 2 & - & - \\
\hline Guyana & 1 & - & - & - \\
\hline Haiti & 3 & - & - & - \\
\hline Honduras & 1 & 1 & - & - \\
\hline Mexico & 101 & 11 & 5 & 13 \\
\hline Nicaragua & 1 & 1 & - & - \\
\hline Panama & 78 & - & - & 1 \\
\hline Paraguay & 2 & - & - & - \\
\hline Peru & 49 & 2 & - & 13 \\
\hline Suriname & 15 & 6 & 1 & 1 \\
\hline $\begin{array}{l}\text { Trinidad and } \\
\text { Tobago }\end{array}$ & 7 & - & - & 2 \\
\hline USA & 377 & 34 & 6 & 173 \\
\hline Venezuela & 92 & 4 & 3 & 3 \\
\hline Total & 1509 & 88 & 42 & 369 \\
\hline
\end{tabular}

LST was selected once more (fourth: phase of the biannual cycle). Aside from precipitation and LST, the only other two variables selected within the top ten list were elevation (DEM) at seventh place with a relative influence of only $3.61 \%$ and MIR (mean), listed tenth and with a relative influence of $3.04 \%$. Land use appears to have little effect, with Globcover channel 160 (closed to open $(>15 \%)$ broadleaved forest regularly flooded (semi-permanently or temporarily), fresh or brackish water) only listed as the thirteenth variable with a relative influence of $1.87 \%$.

The variables selected by the BRT correspond well to the bionomics of Anopheles darlingi. Larvae of this species are most often found in slow flowing rivers, associated with floating debris [72-74]. Rain will impact on river current and height, potentially flushing away the larvae and the river debris they inhabit or causing them to become stranded once the rain lessens or stops and the river recedes $[73,75]$. Land Surface Temperature does not have such a clear cut impact on An. darlingi and could be influencing its distribution in a number of ways. For example, it may be indicating the presence of hotter, dryer environments away from the forests, such as those that limit the distribution of An. darlingi in the north east of its range. Alternatively, LST may be identifying the higher temperatures associated with its lowland range, although there is recent evidence that this species is able to survive at higher altitudes than previously suspected (see below).

\section{Bionomics \\ Anopheles albimanus}

The larval sites used by An. albimanus are characterised across its range as open, sunlit and containing clear water [76] (Table 3). The species can be found in natural and man-made habitats where these characteristics exist. For example, it occurs in recently planted rice fields, or in older fields with sunlit areas in between the rice plants [77] (Table 4). Anopheles albimanus has been associated with floating mats of blue-green algae $[76,78,79]$, which are often found in sunlit waters. The larvae of this ubiquitous species tolerate a wide variation in water chemistry and are able to exploit diverse food sources [78], enabling them to survive in both fresh water (e.g. irrigation channels, small ponds, marshes, slow flowing streams and river margins $[73,80-83])$ and brackish water (e.g. mangrove swamps $[78,83,84])$ (Tables 3-5).

Anopheles albimanus is predominantly exophagic with exophilic resting behaviour $[77,80,85,86]$ (Table 6), however there is some indication that in the northern reaches of its distribution (Mexico, Central America), this species exhibits a preference for resting indoors after feeding $[87,88]$. In a mark-recapture study that ultimately influenced changes in the vector control regime in southern Mexico [87], Bown et al. [89] examined An. albimanus resting behaviour and found that $80 \%$ landed indoors after feeding. Anopheles albimanus bites in the evening and during the night $[77,80,85,86,90,91]$. It appears to show a tendency for zoophily, but this is dependent upon location $[86,89,91,92]$. In Colombia, Solarte et al. [86] described An. albimanus as exhibiting "a high degree of anthropophilic activity". In contrast, Loyola et al. [92] described this species as highly zoophilic in Mexico, however they also pointed out that host availability and other ecological conditions influence the host choice of this species.

\section{Anopheles albitarsis complex}

The An. albitarsis species complex includes An. albitarsis (formerly Sp. A), An. albitarsis Sp. B and Sp. E, An. 
Table 8 Georeferenced and non-georeferenced occurrence data by species and area type: points $\left(\leq 10 \mathrm{~km}^{2}\right)$, wide areas $\left(10-25 \mathrm{~km}^{2}\right)$, small $\left(25-100 \mathrm{~km}^{2}\right)$ and large $\left(>100 \mathrm{~km}^{2}\right)$ polygons, for the nine American DVS (geographically independent sites (Site) and temporal independent occurrences (Occ))

\begin{tabular}{|c|c|c|c|c|c|c|c|c|c|c|}
\hline \multirow[b]{3}{*}{ Species } & \multicolumn{10}{|c|}{ Georeferenced } \\
\hline & \multicolumn{2}{|c|}{ Point } & \multicolumn{2}{|c|}{ Wide-area } & \multicolumn{2}{|c|}{ Small polygon } & \multicolumn{2}{|c|}{ Large polygon } & \multicolumn{2}{|c|}{ All sites } \\
\hline & Site & Occ & Site & Occ & Site & Occ & Site & Occ & Site & Occ \\
\hline An. albimanus & 433 & 822 & 12 & 29 & 7 & 14 & 28 & 38 & 480 & 903 \\
\hline An. albitarsis & 164 & 482 & 5 & 33 & 13 & 48 & 126 & 131 & 308 & 694 \\
\hline An. aquasalis & 86 & 162 & 2 & 17 & 10 & 28 & 28 & 28 & 126 & 235 \\
\hline An. darlingi & 488 & 859 & 25 & 67 & 15 & 106 & 124 & 153 & 652 & 1185 \\
\hline An. freeborni & 31 & 35 & 7 & 28 & 1 & 1 & 5 & 6 & 44 & 70 \\
\hline An. marajoara & 55 & 91 & 4 & 4 & 3 & 4 & 7 & 22 & 69 & 121 \\
\hline An. nuneztovari & 199 & 433 & 11 & 34 & 14 & 14 & 116 & 121 & 340 & 602 \\
\hline An. pseudopunctipennis & 285 & 454 & 7 & 19 & 6 & 6 & 15 & 16 & 313 & 495 \\
\hline An. quadrimaculatus & 356 & 542 & 27 & 30 & 6 & 22 & 168 & 509 & 557 & 1103 \\
\hline \multirow[t]{3}{*}{ Total } & 2097 & 3880 & 100 & 261 & 75 & 243 & 617 & 1024 & 2889 & 5408 \\
\hline & \multicolumn{10}{|c|}{ Non-georeferenced } \\
\hline & \multicolumn{2}{|c|}{ Point } & \multicolumn{2}{|c|}{ Wide-area } & \multicolumn{2}{|c|}{ Small polygon } & \multicolumn{2}{|c|}{ Large polygon } & \multicolumn{2}{|c|}{ All sites } \\
\hline Species & Site & Occ & Site & Occ & Site & Occ & Site & Occ & site & Occ \\
\hline An. albimanus & 44 & 109 & 5 & 7 & 2 & 2 & 2 & 2 & 55 & 125 \\
\hline An. albitarsis & 24 & 28 & 0 & 0 & 0 & 0 & 1 & 1 & 25 & 29 \\
\hline An. aquasalis & 0 & 0 & 0 & 0 & 0 & 0 & 1 & 1 & 1 & 1 \\
\hline An. darlingi & 117 & 120 & 0 & 0 & 1 & 1 & 1 & 1 & 122 & 132 \\
\hline An. freeborni & 0 & 0 & 0 & 0 & 0 & 0 & 0 & 0 & 0 & 0 \\
\hline An. marajoara & 7 & 7 & 0 & 0 & 0 & 0 & 0 & 0 & 7 & 7 \\
\hline An. nuneztovari & 93 & 93 & 0 & 0 & 0 & 0 & 1 & 1 & 96 & 97 \\
\hline An. pseudopunctipennis & 29 & 32 & 6 & 42 & 1 & 1 & 2 & 2 & 38 & 77 \\
\hline An. quadrimaculatus & 21 & 63 & 2 & 2 & 0 & 0 & 0 & 0 & 24 & 66 \\
\hline Total & 335 & 452 & 13 & 51 & 4 & 4 & 8 & 8 & 368 & 534 \\
\hline
\end{tabular}

marajoara (formerly Sp. C) and An. deaneorum (formerly Sp. D). Anopheles marajoara is discussed separately below. Overall, members of this complex exhibit larval habitat preferences similar to $A n$. albimanus, occupying sunlit, clear, fresh water [93,94] (Rubio-Palis, unpub. obs.) (Table 3), although there are examples of it being found in atypical conditions, Da Silva-Vasconcelos et al. [95] found relatively high numbers, compared to other species, in brick pits containing turbid water mixed with clay particles [95]. Typical habitats are fresh, still water bodies such as lagoons, lakes or rice fields [74,93,96] (Table 4). Indeed, members of the An. albitarsis complex are closely associated with human rice cultivation [97,98], although, like An. albimanus, immature stages tend to be found only in fields with early stages of rice growth [98]. Adults are generally exophilic in their resting behaviour $[99,100]$, but will bite both indoors and outdoors and appear to show little host preference, biting humans and animals indiscriminately (dependent on location), during the evening and at night [95,99-105] (Table 6).

\section{Anopheles aquasalis}

Grillet [106] described An. aquasalis as an opportunistic species whose individuals "may be poor competitors or may develop few anti-predator defences". In areas where An. aquasalis and An. albimanus co-exist, An. albimanus will dominate (Rubio-Palis, unpub. obs.) suggesting that the inability of $A n$. aquasalis to out-compete other dominant Anopheles species may be highly influential in defining its ecological requirements (as its name implies, it is generally only found in coastal areas).

Anopheles aquasalis is found in sunlit habitats containing emergent vegetation, in both brackish and fresh water $[93,106,107]$ (Table 3). It is considered to "prefer" clear, still, non-polluted water such as stream pools, mangrove swamps, grass swamps, lagoons and ditches $[108,109]$, although there are examples of it being found in turbid, slow flowing water bodies, in relatively high numbers (e.g. [93]) (Tables 4 \&5).

Adults, once again, are opportunistic, feeding indoors or outdoors on animal or human hosts, but generally resting outdoors before and after feeding [100,110-112]. 
Table 9 Evaluation statistics and the top five environmental/climatic variables selected by the BRT for the nine DVS in the Americas using presence data plus 500 pseudo-presences generated from within the EO boundary (weight $=0.5$ ) and a $1000 \mathrm{~km}$ buffer area for generation of 5:1 pseudo-absence:presence at a $5 \mathrm{~km}$ resolution

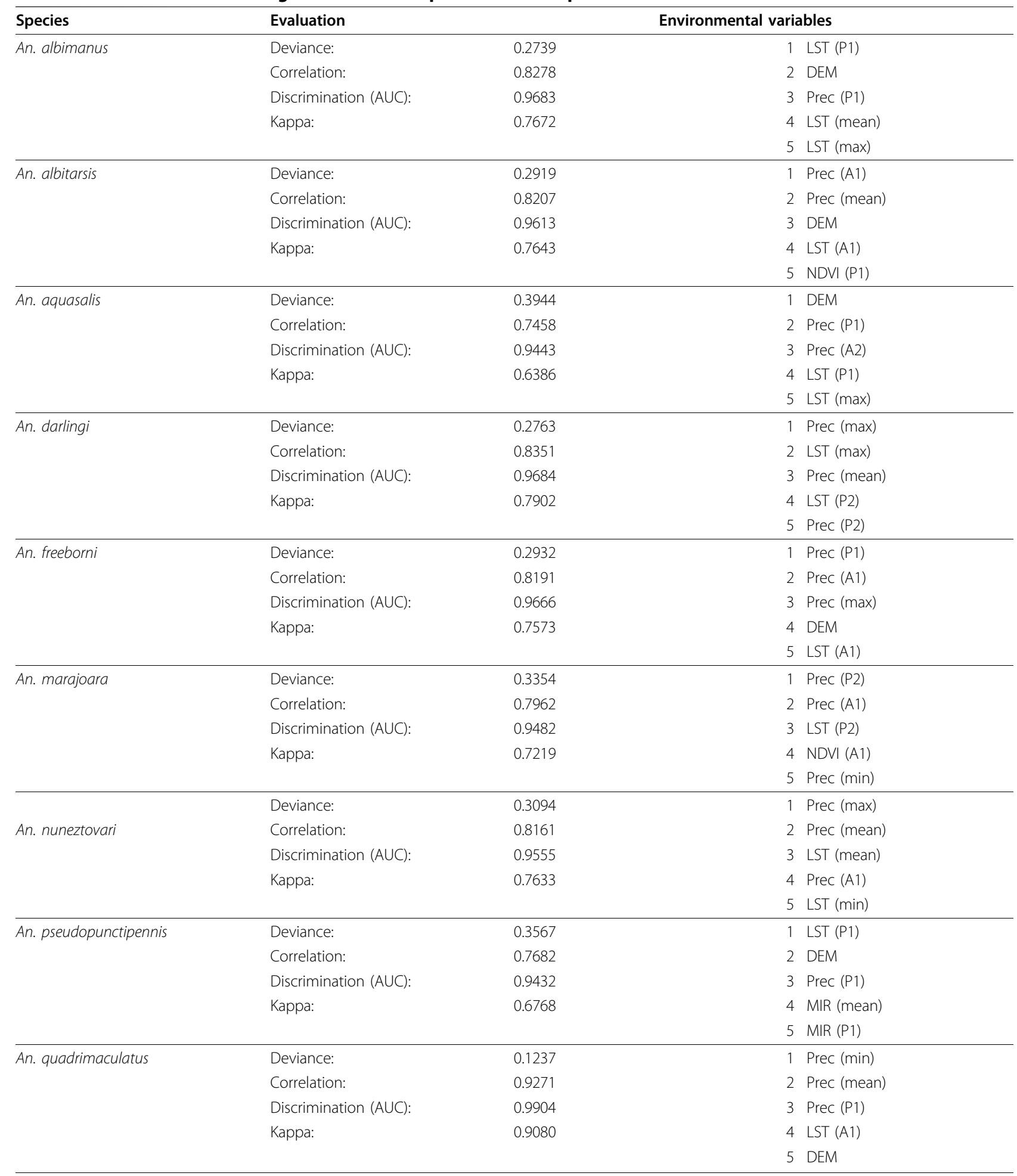




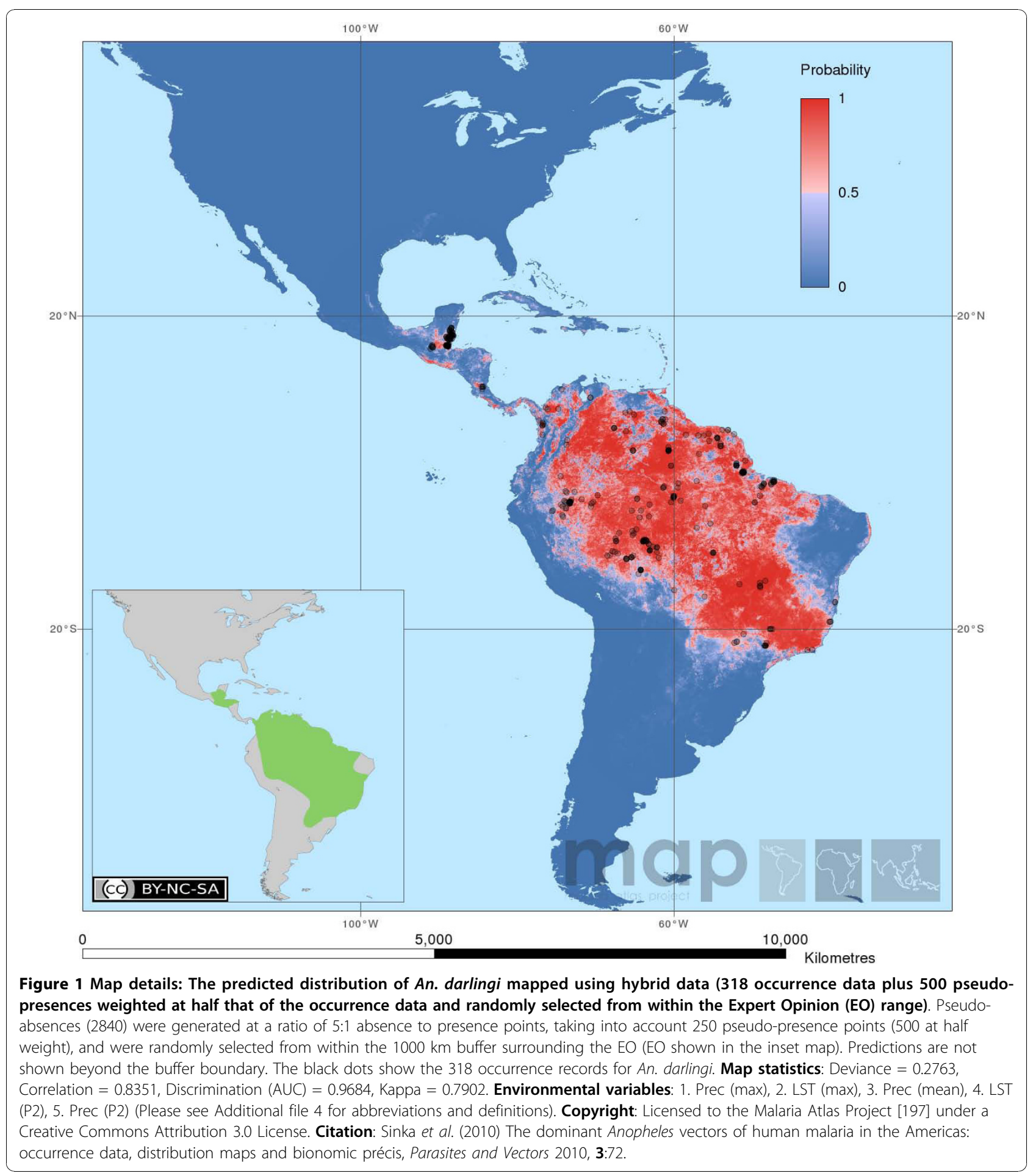

Biting tends to begin at dusk, peaking early in the night, and tailing off as the night progresses [111,112] (Table 6). The time of the biting peak and bias towards endoor exophagy depends on the location, for example in Maranhão, Brazil, Xavier \& Rebelo [111] observed a tendency to bite at dusk, indoors. Berti et al. [112] measured biting behaviour in two villages in Sucre State, Venezuela, where $A n$. aquasalis predominantly bit outdoors, and found that biting peaked earlier $(7 \mathrm{pm})$ in the village of Guayana, where greater numbers of $A n$. 
aquasalis were found, than in Santa Fe, where the peak occurred between 8 and $9 \mathrm{pm}$. During the study, two females were collected biting during the day.

\section{Anopheles darlingi}

Anopheles darlingi is considered to be one of the most efficient malaria vectors in the Neotropical region [23]. It is mainly a riverine mosquito, generally confined to rural, lowland forested locations [113] (Rubio-Palis \& Manguin, unpub. obs.). Conversely, however, Vittor et al. [114] suggest that deforestation and human environmental alteration can create habitats which are favourable to An. darlingi as they found higher densities in areas with limited forest cover than in areas predominated by forest. The larval habitats of An. darlingi can be characterized as: natural water bodies such as lagoons, lakes and particularly slow flowing streams or rivers with shaded, clear water, and associated submersed vegetation such as bamboo roots from overhanging spiny bamboo [72-74,113] (Tables 3-5). Larvae are encountered most frequently in patches of floating debris along river margins [73]. There are examples of larvae being found in uncharacteristic locations, however, such as in slightly brackish water (Belize, [73]), in low numbers in turbid, polluted water (brick pits, [95]) and in abandoned gold mine dugouts in southern Venezuela [115], further suggesting a level of adaptation to areas altered by humans [113].

Anopheles darlingi tends to rest outdoors regardless of where it has taken its blood meal [116,117] (Table 6). Adults will bite throughout the night [95,117-120] and the degree of endo- and exophagy of this species varies from one place to another as does its host preference [121] (Table 6). It has been suggested that the biting pattern of An. darlingi may represent an adaptation to human behaviour $[117,121]$. Moreno et al. [117], for example, contend that the all night activity of An. darlingi in the gold mining areas of southern Venezuela is a response to the all night activity of the miners. Furthermore, a number of studies that report exophagy in this species (Table 6) refer to peri-domestic sampling $[113,116,122]$ at sites where indoor insecticide spraying is or had recently been used for vector control $[113,117,122,123]$. Other indications of the adaptability of $A n$. darlingi are emerging, with an ongoing study in Venezuela, close to the Brazilian border (Roraima), recently reporting that specimens have been collected at altitudes above $800 \mathrm{~m}$ (Rubio-Palis, unpub. obs: re. Berti et al. at the Instituto de Altos Estudios "Dr. Arnoldo Gabaldon", Ministry of Health).

\section{Anopheles freeborni}

Studies examining the bionomics of An. freeborni tend to have been performed prior to 1985 limiting the amount of useful data found within the MAP library. Therefore additional searches of older references were needed to develop a more complete picture of the bionomics of this species.

McHugh [124] suggests that An. freeborni is an opportunistic feeder. In a study where its blood meals were identified, host availability was key in determining blood source. For example, the An. freeborni females collected in cattle areas contained the highest proportion of bovine blood, whereas $35 \%$ had fed on dogs at sites of human habitation near a rice production area, and no bovine blood was found. This suggests that the species is zoophilic, although a preference is not indicated in this study. Washino \& Tempelis [125], in a similar study, reported very low levels of human blood in $A n$. freeborni $(<1 \%$ of mosquitoes tested) even though sampling was conducted in areas of human habitation. They also indicated the opportunistic nature of this species, given the high level of rabbit blood meals found. However, once again, the proportion was highly dependent on rabbit availability. The resting mosquitoes were collected from farmyard sheds, abandoned domestic animal shelters, house porches, artificial shelters, bridges and culverts, indicating an outdoor resting preference.

Orr \& Resh [126] demonstrated a positive association between An. freeborni larval densities and plant cover, indicating that, as with all the other DVS in the Americas, vegetation is a key characteristic of their larval habitats. This is reflected by the species' ready utilization of rice fields, although significantly higher numbers of adult $A n$. freeborni are found in riparian and mixed habitats than in rice and pasture habitats $[127,128]$ (Table 4).

\section{Anopheles marajoara}

Anopheles marajoara is a member of the An. albitarsis complex. Previously believed to be a secondary, local vector of minor importance, it was identified as a DVS in the 1990s in a study conducted in Amapa, Brazil [129], where it was found in high densities and with high levels of Plasmodium infection when compared to An. darlingi. Here, therefore, this species is considered separately from other members of the An. albitarsis complex.

Anopheles marajoara is a lowland species, associated with wetlands, secondary forests and human intervention [115,129] (Rubio-Palis, unpub. obs.). Moreno et al. [117] suggest that the recent studies in Brazil identifying An. marajoara as dominant over An. darlingi may be a result of human interventions which favour species that oviposit in open lagoons with abundant macrophytes. Conn et al. [129] described forest clearance and pollution as reducing the availability of larval sites for $A n$. darlingi whilst increasing the availability of sites such as agricultural ponds and sunlit marshy areas, which are the preferred habitats of An. marajoara. Overall, $A n$. marajoara larval sites are generally sunlit with clear, 
still water, although there are examples of this species being found in both clear and muddy waters, such as fish ponds [113] and gold mine dugouts [115] (Tables 35).

In Amapa, An. marajoara is described as exclusively exophilic [129], which appears to be the case across its range $[99,116,117]$ (Rubio-Palis, unpub. obs.), however a study in Colombia examining the indoor resting behaviour of anophelines, reported An. marajoara resting indoors, close to the ground [130], suggesting some endophilic behaviour in limited areas (Table 6).

Anopheles marajoara bites both humans and animals $[102,116,131]$, both indoors and outdoors throughout the night, with biting tending to peak in the evening $[99,113,117,129]$ (Table 6), though again these characteristics can vary according to location. For example, a study in southern Venezuela reported marked exophagic behaviour, with $74.2 \%$ of An. marajoara captured biting outdoors [117]. This contrasts with a study carried out in western Venezuela [99] that reported indiscriminate indoor and outdoor biting. There is currently some debate, however, about the identity of members of the An. albitarsis complex in Venezuela. Past investigations have only identified the presence of An. marajoara $[117,132]$, but a recent study suggests the possibility of misidentification and the additional presence of An. janconnae [133]. A consensus for this "discovery" has yet to be reached.

\section{Anopheles nuneztovari}

The An. nuneztovari species complex is yet to be fully resolved and there is a need for some clarification of sibling identity. The complex contains either two or three cytological species (A and $B / C$ ), with $B$ and $C$ possibly two forms of a single species [134-137] (RubioPalis \& Manguin, unpub. obs.). Furthermore, Calado et al. [137] have recently formally resurrected An. goeldii from synonymy with An. nuneztovari A, making the task of truly categorising members of this complex all the more challenging.

Anopheles nuneztovari larvae are found in both sunlit and shaded habitats [74,138] (Rubio-Palis, unpub. obs.). Sites usually contain fresh, clear, still or flowing water with floating or emergent vegetation [74,138] (Table 3). Despite this, Nagm et al. [74] report finding this species in several turbid water bodies, and da Silva-Vasconcelos et al. [95] found small numbers in brick pits containing very turbid water polluted with brick dust. Indeed, Service [28] described An. nuneztovari larval habitats as "muddy waters of pools, vehicle tracks, hoof prints, small ponds, especially in and around towns".

Habitats are found in small or large, natural or constructed bodies of water, including lagoons, lakes, slow flowing rivers, fish ponds, gold mine dugouts, rain puddles and temporary or permanent pools [80,138-140]
(Table 4 \&5). Tadei \& Thatcher [141] described An. nuneztovari as a species readily able to colonise and even dominate in altered environments and yet, despite this characteristic, it is not known to breed in rice fields.

Adult behaviour differs depending on the sibling species, most specifically in terms of their biting times, with An. nuneztovari A (Brazil) biting earlier, peaking between 6 and 8 pm [95,141,142], and An. nuneztovari $\mathrm{B} / \mathrm{C}$ (Venezuela and Colombia) biting later and throughout the night, peaking between $10 \mathrm{pm}$ and 2 am [142]. Olano et al. [80] reported peri-domiciliary biting of $A n$. nuneztovari early in the evening in Buenaventura, Colombia, however this may be the result of low biting densities rather than a true indication of preference. The Brazilian sibling is considered to be a non-vector, possibly due to its behaviour rather than of an inability to transmit malaria $[142,143]$, given that there is evidence of Plasmodium infection in An. nuneztovari in Amapa, Brazil [144,145].

Service [28] suggested that An. nuneztovari mainly feeds on animals, but will bite humans outdoors. Studies in Amapa, Brazil and in Venezuela, which analysed blood meals of An. nuneztovari, suggest zoophilic behaviour, but an accompanying human landing catch in the same area found over 120 mosquitoes/person/night $[99,102,116]$. Exo- and endophagy of members of this species complex vary with location but Tadei \& Thatcher [141] suggested that human behaviour, such as a propensity to stay outdoors late into the evening, or the application of insecticides, may influence biting location. The majority of studies summarised here report exophagic behaviour $[80,99,116,139,141]$ (Table 6), with only Rubio-Palis \& Curtis [99] reporting both endo- and exophagic behaviour in western Venezuela. Rubio-Palis \& Curtis [99] mentioned the contrasting observations of exo- and endophagy reported for An. nuneztovari and suggested this may be due to collection bias (collector, location or short series of observations) or a result of different behavioural patterns between sympatric siblings, which again highlights a need for further clarification of species identity. All sibling species within the An. nuneztovari complex are highly exophilic, resting outdoors both before and after feeding $[99,116]$ (Table 6).

\section{Anopheles pseudopunctipennis}

Anopheles pseudopunctipennis is a complex of at least two species [146,147] and two forms [148] (Rubio-Palis \& Manguin, unpub. obs.). It can survive and transmit malaria at altitudes higher than many other DVS, with its range extending up to approximately $3000 \mathrm{~m}$ [22,149] (Rubio-Palis \& Manguin, unpub. obs.). This species is most frequently found in sun-exposed, shallow, clear and freshwater streams or river pools with abundant filamentous algae [149], although there are a 
number of reports of larvae found in turbid, cloudy water $[93,109,149]$, including at one site polluted with cow faeces [149]. The majority of larval habitats have fresh water, but about $10 \%$ contain brackish or sea water [93,149] (Table 3). Past studies conducted in Grenada suggested that An. pseudopunctipennis was restricted to still or stagnant water [150] but more recent investigations on this island indicate that it can survive in slow flowing water bodies, possibly protected against the current by mats of Spirogyra-type green filamentous algae $[109,149]$. The presence of such filamentous algae is a key characteristic associated with larval habitats of this species [93,109,149,151-154] (Table 3). Indeed, a study that examined the potential impact of An. pseudopunctipennis control via environmental manipulation demonstrated significant reductions in densities after the removal of filamentous algae from larval sites [151].

Service [28] stated that adult An. pseudopunctipennis "...feed almost indiscriminately on humans and domestic animals, indoors or outdoors...". Studies conducted in southern Mexico corroborate his statement, for example Fernandez-Salas et al. [155] found that a greater proportion of An. pseudopunctipennis were attracted to horsebaited traps than to humans (although significant numbers were captured on humans), however in a previous study in the same four villages [156], they found that a high proportion of the An. pseudopunctipennis females resting indoors contained human blood. They suggested that host availability was responsible for host selection, rather than this being due to any preference exhibited by the mosquitoes. Lardeux et al. [157], in a study specifically designed to examine host preference, found some level of choice exhibited by females. They suggested that this species is not highly anthropophilic, but reiterated its opportunistic nature, stating that $A n$. pseudopunctipennis females will bite the first "preferred" host they encounter (in their experiment the feeding preference was ranked as: sheep, goats and donkeys, followed by humans and cows).

Service [28] also stated that "[An. pseudopunctipennis] rest outdoors after feeding", however the studies above $[155,156]$, and those of Casas et al. [158], who used the mark-recapture method to identify resting behaviour of fed and unfed mosquitoes, indicated that a proportion of An. pseudopunctipennis will rest indoors both before and after feeding. However, Loyola et al. [159] suggested that IRS with DDT has not only increased insecticide resistance in some areas, but also promoted more exophilic behaviour.

Anopheles pseudopunctipennis bites during the night, with small variations in peak activity depending on location and host, for example Fernandez-Salas et al. [155] demonstrated a uni-modal peak, with indoor biting peaking at 1 am and outdoor biting peaking slightly earlier, at midnight. Interestingly, they also reported a bimodal biting pattern on horse bait, where biting peaked at $7 \mathrm{pm}$, with a second, smaller peak occurring between midnight and 1 am (Table 6).

\section{Anopheles quadrimaculatus subgroup}

The Quadrimaculatus Subgroup of the Maculipennis Group is often mis-reported as a complex (e.g. $[19,69,70])$. Within this subgroup, An. quadrimaculatus (formerly Sp. A) is the most wide-spread species [19,70], considered the most "important" [70], and it is the only species within the subgroup identified as a DVS [25-29]. Therefore only this species is given further consideration here, with the caveat that some studies included in the bionomics review do not distinguish species and report An. quadrimaculatus s.l. However, as suggested by Seawright et al. [70], owing to its abundance, seconded only by An. smaragdinus (formerly An. quadrimaculatus Sp. B), An. quadrimaculatus (and An. smaragdinus) "are probably the species that most researchers have studied in the past".

Anopheles quadrimaculatus is highly associated with rice cultivation [69,160-166] (Table 4), showing a preference for the oligotrophic conditions found when the rice fields are first flooded [162]. Such conditions reflect those found in the natural larval habitat of this species: generally fresh, still water in relatively large sites such as lakes and marshes with emergent vegetation [69,167-170] (Tables 3 \&4). Unusual larval sites have been reported including a sewerage retention pond containing highly polluted effluent draining from a pig farm, a small plastic bucket containing rainwater, submerged leaf litter and floating pine needles [171], and wastewater evaporation-percolation ponds in Florida [172] (Table 5).

Adults appear to be generally zoophilic, biting and resting outdoors $[69,168,170]$ (Table 6), however, amongst all studies summarised, this may be an artefact of the areas sampled and the lack of human hosts rather than an indication of preference $[168,170]$. Of the species in this subgroup, however, An. quadrimaculatus appears to show the highest level of anthropophily. Jensen et al. [168] examined blood meals in An. quadrimaculatus (Sp. A), Sp. B (An. smaragdinus) and Sp. $\mathrm{C}_{1}$ (An. diluvialis) at a campsite where human hosts were available, and in a woodland approximately a mile away, where there was a lower chance of human contact, and found that $10.7 \%$ of An. quadrimaculatus sampled at the campsite had fed on humans compared to none in the wooded area. The other two species demonstrated very low or no human biting at both sites. Reinert et al. [69], in a comprehensive description of the taxonomy and bionomics of the Quadrimaculatus Subgroup, also reported such exophagic and zoophilic behaviour, 
describing frequent collections of engorged females from horse stables and cattle barns and a number of observations of feeding on large domestic animals.

Anopheles quadrimaculatus bites throughout the night, showing higher activity at dusk and dawn [69]. Resting behaviour, as with all other DVS described here, is exophilic, and includes sites in holes and rot cavities in trees, livestock barns, outdoor latrines, under bridges and under the eaves of buildings $[69,170,173,174]$ (Table 6).

\section{Secondary vectors}

This study has focused on describing the distribution and bionomics of the main malaria vectors in the Americas. However, there are numerous secondary or local vectors that may also play an important yet often forgotten role in malaria transmission. In the Americas, secondary vectors include the various species of the subgenus Kerteszia (e.g. An. cruzii, An. bellator and An. neivai), whose larvae characteristically inhabit water contained in bromeliads, as well as An. (Anopheles) vestitipennis, An. (Ano.) neomaculipalpus, An. (Nyssorhynchus) braziliensis, An. (Nys.) triannulatus, An. (Nys.) strodei, An. (Ano.) intermedius and members of the An. (Nys.) oswaldoi complex. Their "secondary" vector status is a factor of location, distribution, vectorial capacity and, occasionally, history (i.e. a species once considered primary, but now relegated to a secondary role through changes in the local environment). Circumstantial evidence, or the identification of Plasmodium circumsporozoite proteins in females, can lead to a species being incriminated as a vector, where in reality it may have little to no impact [23]. However, some "secondary" species are proven and potent vectors within their local range.

Both An. cruzii and An. bellator are identified by White [29] as main malaria vectors, yet their larval habitats (bromeliads) restrict their role in malaria transmission to areas where such plants are abundant, for example in the rainforests of Brazil where these two species are considered to be primary local vectors. Deforestation may have reduced the availability of habitats for these species but they ought not to be overlooked as their range extends along the eastern coast of South America, from Guyana to the southernmost tip of Brazil. Moreover, there are reports of these species being found in bromeliads and artificial containers in urban and peri-urban sites $[175,176]$ with An. cruzii specifically described as an aggressive biter throughout the day and night [177]. Anopheles neivai also makes use of bromeliads during its larval stage and is an important vector of human malaria in the Pacific coastal areas of Colombia $[178,179]$. It has been responsible for outbreaks of malaria in the Venezuelan Andes at altitudes above $1000 \mathrm{~m}[180,181]$.
Anopheles vestitipennis is considered to be a secondary vector species of major importance within its range. In Belize, it has been described as a primary vector, ousting An. albimanus which plays a more secondary role there [182-185]. Achee et al. [183] found it to be positive for both $P$. falciparum and $P$. vivax and Loyola et al. [186] found it to be the most abundant species ( $>80 \%$ of those collected on human bait) in their study area within the Lacandon rainforest of Chiapas, Mexico, and the only species in the area to be positive for the $P$. vivax antigen. They also demonstrated that it readily bites humans both indoors and outdoors. Anopheles vestitipennis is also found in the Caribbean islands where it may have been involved in malaria outbreaks in Cuba [187] and Haiti (Rubio-Palis, unpub. obs.).

Malaria parasites have also been detected in several other species. De Oliveira Ferreira et al. [188] found natural infections of Plasmodium in An. triannulatus, An. braziliensis, An. strodei and An. oswaldoi in Rondonia, Brazil. Anopheles triannulatus and An. strodei were only infected with $P$. vivax, however the very small sample sizes (the largest being five specimens of An. triannulatus), prevents the drawing of any conclusions regarding any refractory characteristics.

Anopheles oswaldo $i$ has been confirmed as a malaria vector in Brazil [119,188-190], and it is the principal vector in the State of Acre in the Brazilian Amazon where it has been found in large densities and with relatively high sporozoite rates for $P$. falciparum (3.41\%), $P$. vivax-210 (2.26\%), P. vivax-247 (1.22\%) and P. malariae $(0.42 \%)$ [189]. Anopheles oswaldoi has also been incriminated as a vector of P. vivax in Colombia [191], Peru [192] and Venezuela [193].

The role of An. neomaculipalpus in malaria transmission is not clear but it has recently been found to be positive for P. vivax in Venezuela $[194,195]$ and positive for P. falciparum in Colombia [195,196] and it is considered to be a highly anthropophilic species ([195]). Moreno et al.[195] suggested that this species may play a role as a secondary vector of "frontier malaria" in areas of forest subject to recent human activity that can increase vector diversity with the creation of new larval sites. De Oliviera Ferreira et al. [188] reiterated this hypothesis and suggested that the presence of infection amongst these secondary vectors may be linked to the extensive environmental changes that may reduce the populations of sylvatic animals in an area whilst simultaneously increasing the number of humans, which will inevitably cause a higher level of vector-human contact.

\section{Discussion}

\section{Reliability of the predictive maps}

The predictive maps presented here are unlikely to be perfect representations of the full distributions of the 
DVS. The model has been applied to species presence data from one of the largest, most comprehensive, contemporary databases of DVS occurrence available, yet the limitations of these opportunistic data for species mapping are evident. Biases in collection location, limited data for some species and a lack of consistency in sampling methodology all contribute to modelling and output uncertainty. Despite these caveats, the maps represent the first attempt to model DVS distributions in the Americas using extensive occurrence data, contemporary EO range maps and a consistent methodology across all species. Previous maps have either focussed on a single species or subgroup [19], single countries $[20,21]$, been based solely on EO [22,23], or, specimens collected over a large period of time [18]. The maps are best considered a starting point in a continuing process of describing and understanding DVS distributions, and not as an end product. Therefore, and in accordance with the open access principles of the MAP, all the data compiled here will be made freely available on the MAP website [197] and efforts made to improve the maps as more data become available.

\section{Bionomics}

The bionomics review highlights DVS behaviour and life-history characteristics that are relevant for mosquito control, but also clearly indicates the marked behavioural plasticity of each species. The influence of human behaviour such as insecticide use, environmental disturbance to a greater or lesser extent, or host activities in the evening and night also drive local variation in species bionomics. Moreover, concerns regarding species identity also add to the uncertainty in categorising species behaviour and thus local, expert knowledge must be consulted when interpreting or acting on the data summarised here.

\section{Future work}

This is the first in a series of three publications describing the distribution and relevant bionomics of the global DVS of $P$. falciparum. The remaining two publications will detail the DVS of Africa, Europe and the Middle-East (Sinka et al: The dominant Anopheles vectors of human malaria in Africa, Europe and the Middle East: occurrence data, distribution maps and bionomic précis, unpublished), and the DVS of the Asian Pacific region (Sinka et al: The dominant Anopheles vectors of human malaria in the Asia Pacific region: occurrence data, distribution maps and bionomic précis, unpublished). Together, these three publications are intended to provide a baseline set of data and maps and summarise the current knowledge of the bionomics of the 41 species (DVS) identified as the primary vectors of $P$. falciparum and $P$. vivax malaria.

\section{Conclusions}

Species distribution mapping is a dynamic process. The advent of new modelling techniques [47], high resolution climatic and environmental spatial data at local and global scales [52-55,57,58,198], increasing computer processing capacity and a greater on-the-ground knowledge of what drives and limits the range of a species provide the tools for increasingly accurate map production. These maps are often limited by the data available to the model rather than the modelling process itself. Increasing openness and a willingness to share data across disciplines and groups will prevent duplication of effort in data-rich areas, and encourage the development of systematic vector sampling procedures in areas where the information is relatively poor.

\section{Additional material}

Additional file 1: Expert opinion distribution maps for the nine DVS of the Americas.

Additional file 2: Predictive species distribution maps for the nine DVS of the Americas.

Additional file 3: Bionomics protocol.

Additional file 4: Summary tables showing evaluation statistics for all mapping trials and final Boosted Regression Tree environmental and climatic variable selections for the final, optimal predictive maps.

\section{List of abbreviations}

DVS: Dominant Vector Species; BRT: Boosted Regression Trees; IVM: Integrated Vector Management; MIMP: Mosquito Information Management Project; TAG: Technical Advisory Group; MAP: Malaria Atlas Project; WRBU: Walter Reed Biosystematics Unit; EO: Expert Opinion; GIS: Geographic Information System; PCR: Polymerase Chain Reaction; ITNs: Insecticide Treated Bednets; IRS: Insecticide Residual Spraying; MES: Marianne E; Sinka AUC: Area Under the operating characteristic Curve; TFA: Temporal Fourier Analysis; SRTM: Shuttle Radar Topography Mission; DEM: Digital Elevation Model; MODIS: MODerate Resolution Imaging Spectroradiometer; NASA: National Aeronautics and Space Administration; AVHRR: Advanced Very High Resolution Radiometer; NDVI: Normalized Difference Vegetation Index; LST: Land Surface Temperature; MIR: Middle Infrared Radiation; MERIS: Medium Resolution Imaging Spectrometer; MBO: Human bait landing catches outdoors ('Man-Biting Outdoors'); IVCC: Innovative Vector Control Consortium.

\section{Acknowledgements}

We wish to thank Philip Mbithi, Robi Okara, Rosalind Howes, Edward Haynes, Owen Yang, Carolynn Tago, and Elisabeth Thiveyrat for primary data abstraction and Anja Bibby and Rosalind Howes for proof-reading the manuscript. We also thank the Technical Advisory Group for extended support over the duration of the project (in addition to co-authors Ralph Harbach, Sylvie Manguin and Yasmin Rubio-Palis and these include Mike Bangs, Theeraphap Chareonviriyaphap, Janet Hemingway and Charles M. Mbogo). The following persons provided valuable unpublished information for the malaria vectors in the Americas: Marylin Aparicio (Bolivia), Mauricio Casas (Mexico), Roberto Fernández (Peru), Ranulfo González (Colombia), and Ricardo Lourenço-de-Oliveira (Brazil). MES is funded by a project grant from the Wellcome Trust (\#083534) to SIH. SIH is funded by a Senior Research Fellowship from the Wellcome Trust (\#079091) which also supports CWK and PWG. APP and WHT are funded by a Wellcome Trust Principal Research Fellowship (\#079080) to Professor Robert Snow. This work forms part of the 
output of the Malaria Atlas Project (MAP, http://www.map.ox.ac.uk), principally funded by the Wellcome Trust, U.K.

\section{Author details}

'Spatial Ecology and Epidemiology Group, Tinbergen Building, Department of Zoology, University of Oxford, South Parks Road, Oxford OX1 3PS, UK. 2BIOMED, Universidad de Carabobo, Apartado 2073, Maracay 2101-A, Venezuela. ${ }^{3}$ Laboratorio de Ecología de Vectores, Dirección de Control de Vectores y Fauna Nociva, Ministerio del Poder Popular para la Salud, Maracay, Venezuela. ${ }^{4}$ Institut de Recherche pour le Développement, Lab. d'Immuno-Physiopathologie Virale et Moleculaire, UMR-MD3/Univ. Montpellier I, Faculté de Pharmacie, 15, Ave Charles Flahault, 34093 Montpellier, France. ${ }^{5}$ Biological Control and Spatial Ecology, Université Libre de Bruxelles CP160/12, Av FD Roosevelt 50, B1050, Brussels, Belgium. ${ }^{6}$ Malaria Public Health and Epidemiology Group, Centre for Geographic Medicine, KEMRI - Univ. Oxford - Wellcome Trust Collaborative Programme, Kenyatta National Hospital Grounds, P.O. Box 43640-00100 Nairobi, Kenya. ${ }^{7}$ Department of Entomology, The Natural History Museum, Cromwell Road, London, UK

\section{Authors' contributions}

$\mathrm{SIH}$ conceived the study and managed its design and implementation. MES and $\mathrm{SIH}$ wrote the first draft of the manuscript, MES assembled the occurrence data with assistance from CWK (also see Acknowledgements), CWK also digitised and edited all the expert opinion maps. WHT designed and maintained the databases and implemented the map figures. APP implemented the BRT scripts for predictive mapping. PWG processed the environmental and climatic data grids, with assistance from TVB. Experiments were derived by SIH and MES and implemented by MES. All authors participated in the interpretation of results and in the writing and editing of the manuscript. YR-P, SM and REH advised on bionomics and nomenclature issues, and provided additional comments and input to the manuscript.

\section{Competing interests}

The authors declare that they have no competing interests.

Received: 6 July 2010 Accepted: 16 August 2010

Published: 16 August 2010

\section{References}

1. Guerra CA, Gikandi PW, Tatem AJ, Noor AM, Smith DL, Hay SI, Snow RW: The limits and intensity of Plasmodium falciparum transmission: implications for malaria control and elimination worldwide. PLoS Med 2008, 5:e38.

2. Hay SI, Guerra CA, Gething PW, Patil AP, Tatem AJ, Noor AM, Kabaria CW Manh BH, Elyazar IR, Brooker S, Smith DL, Moyeed RA, Snow RW: A world malaria map: Plasmodium falciparum endemicity in 2007. PLoS Med 2009, 6:e1000048

3. Gething PW, Patil AP, Hay SI: Quantifying aggregated uncertainty in Plasmodium falciparum malaria prevalence and populations at risk via efficient space-time geostatistical joint simulation. PLoS Comput Biol 2010 6:e1000724.

4. Hay SI, Okiro EA, Gething PW, Patil AP, Tatem AJ, Guerra CA, Snow RW: Estimating the global clinical burden of Plasmodium falciparum malaria in 2007. PLoS Med 2010, 7:e100029.

5. Guerra CA, Howes RE, Patil AP, Gething PW, Van Boeckel TP, Temperley WH, Kabaria CW, Tatem AJ, Manh BH, Elyazar IRF, Baird KJ, Snow RW, Hay SI: The international limits and population at risk of Plasmodium vivax transmission in 2009. PLoS Negl Trop Dis 2010, 4:e774.

6. WHO/PAHO (World Health Organization/Pan American Health Organization) Malaria in the Americas: time series epidemiological data from 2000 to 2007 Washington DC: Pan American Health Organization, Regional Office for the Americas 2008.

7. Roberts DR, Masuoka P, Yu AY: Determinants of malaria in the Americas. In The contextual determinants of malaria. Edited by: Casman EA, Dowlatabadi H. Washington, DC: Resources for the Future Press; 2002:35-58.

8. Rojas W, Botero S, Garcia HI: An integrated malaria control program with community participation on the Pacific Coast of Colombia. Cad Saude Publica 2001, 17(Suppl):103-113.
9. Roberts DR, Laughlin LL, Hsheih P, Legters LJ: DDT, global strategies, and a malaria control crisis in South America. Emerg Infect Dis 1997, 3:295-302.

10. Killeen GF, Fillinger U, Kiche I, Gouagna LC, Knols BG: Eradication of Anopheles gambiae from Brazil: lessons for malaria control in Africa? Lancet Infect Dis 2002, 2:618-627.

11. Butler WP, Roberts DR: Malaria in the Americas: a model of reemergence. Mil Med 2000, 165:897-902.

12. Shiff C: Integrated approach to malaria control. Clin Microbiol Rev 2002, 15:278-293.

13. WHO/PAHO (World Health Organization/Pan American Health Organization): Regional strategic plan for malaria in the Americas 2006-2010 Washington, DC: Pan American Health Organization, Regional Office for the Americas 2006, 71.

14. Snow RW, Guerra CA, Mutheu JJ, Hay SI: International funding for malaria control in relation to populations at risk of stable Plasmodium falciparum transmission. PLoS Med 2008, 5:e142.

15. RBMP (Roll Back Malaria Partnership): The global malaria action plan for a malaria free world Geneva, Switzerland: Roll Back Malaria Partnership, World Health Organization 2008.

16. WHO (World Health Organization): Global strategic framework for integrated vector management Geneva: World Health Organization 2004.

17. WHO (World Health Organization): Guidelines on the elimination of residual foci of malaria transmission Cairo: World Health Organization Regional Office for the Eastern Mediterranean 2007, 47.

18. Foley DH, Weitzman AL, Miller SE, Faran ME, Rueda LM, Wilkerson RC: The value of georeferenced collection records for predicting patterns of mosquito species richness and endemism in the Neotropics. Ecol Entomol 2008, 33:12-23.

19. Levine RS, Peterson AT, Benedict MQ: Distribution of members of Anopheles quadrimaculatus Say s.l. (Diptera: Culicidae) and implications for their roles in malaria transmission in the United States. J Med Entomol 2004, 41:607-613.

20. Loaiza JR, Bermingham E, Scott ME, Rovira JR, Conn JE: Species composition and distribution of adult Anopheles (Diptera: Culicidae) in Panama. J Med Entomol 2008, 45:841-851.

21. Osborn FR, Rubio-Palis Y, Herrera M, Figuera A, Moreno JE: Caracterizacion ecoregional de los vectores de malaria en Venezuela. Bol Mal Salud Amb 2004, XLIV:77-92.

22. Rubio-Palis $\mathrm{Y}$, Zimmerman $\mathrm{RH}$ : Ecoregional classification of malaria vectors in the neotropics. J Med Entomol 1997, 34:499-510.

23. Manguin S, Carnevale P, Mouchet J, Coosemans M, Julvez J, RichardLenoble D, Sircoulon J: Biodiversity of malaria in the world Montrouge, France: John Libbey Eurotext 2008.

24. Hay SI, Sinka ME, Okara RM, Kabaria CW, Mbithi PM, Tago CT, Benz D, Gething PW, Howes RE, Patil AP, Temperley WH, Bangs MJ, Chareonviriyaphap T, Elyazar IRF, Harbach RE, Hemingway J, Manguin S, Mbogo CM, Rubio-Palis Y, Godfray HCJ: Developing global maps of the dominant Anopheles vectors of human malaria. PLoS Med 2010, 7 e1000209.

25. Kiszewski A, Mellinger A, Spielman A, Malaney P, Sachs SE, Sachs J: A global index representing the stability of malaria transmission. Am J Trop Med Hyg 2004, 70:486-498.

26. Mouchet J, Carnevale P, Coosemans M, Julvez J, Manguin S, RichardLenoble D, Sircoulon J: Biodiversité du paludisme dans le monde Montrouge, France: John Libbey Eurotext 2004.

27. Service MW: The Anopheles vector. In Bruce-Chwatt's Essential Malariology. Edited by: Gilles HM, Warrell DA. London: Edward Arnold; , Third 1993:96-123.

28. Service MW: Appendix II. Characteristics of some major Anopheles vectors of human malaria. In Bruce-Chwatt's Essential Malariology. Edited by: Gilles HM, Warrell DA. London: Edward Arnold; Third 1993:305-310.

29. White GB: Malaria. Geographical distribution of arthropod-borne diseases and their principal vectors Geneva: World Health Organization, Division of Vector Biology and Control 1989, 7-22

30. Guerra CA, Hay SI, Lucioparedes LS, Gikandi PW, Tatem AJ, Noor AM, Snow RW: Assembling a global database of malaria parasite prevalence for the Malaria Atlas Project. Malar J 2007, 6:17.

31. PubMed. [http://www.ncbi.n/m.nih.gov/pubmed/].

32. Web of Science. [http://isiwebofknowledge.com/products_tools/ multidisciplinary/webofscience/]. 
33. AnoBase Bibliographical Database. [http://www.anobase.org/cgi-bin/publn. pl].

34. Walter Reed Biosystematics Unit (WRBU), Mosquito Catalog. [http://www. mosquitocatalog.org/default.aspx?pg|D=8].

35. The Disease Vectors Database. [http://www.diseasevectors.org/references php].

36. MalariaWorld database. [http://www.malariaworld.org/].

37. Malaria in the News (Roll Back Malaria) Archives. [http://www rollbackmalaria.org/malariainthenews.html].

38. Environmental Health at USAID (Malaria Bulletin). [http://www.ehproject. org/J.

39. BioMed Central. [http://www.biomedcentral.com/]

40. Vector-Borne and Zoonotic Diseases (Mary Ann Liebert Inc Publishers). [http://www.liebertpub.com/products/product.aspx?pid=67].

41. Indian Journal of Medical Research. [http://www.icmr.nic.in/ijmr/ijmr.htm]

42. MosquitoMap. [http://www.mosquitomap.org/].

43. PostgreSQL Opensource Database. [http://www.postgresql.org/]

44. The Django Project. [http://www.djangoproject.com/].

45. Elith J, Leathwick JR, Hastie T: A working guide to boosted regression trees. J Anim Ecol 2008, 77:802-813.

46. De'ath G: Boosted trees for ecological modeling and prediction. Ecology 2007, 88:243-251.

47. Elith J, Graham CH, Anderson RP, Dudik M, Ferrier S, Guisan A, Hijmans RJ, Huettmann F, Leathwick JR, Lehmann A, Li J, Lohmann LG, Loiselle BA, Manion G, Moritz C, Nakamura M, Nakazawa Y, Overton JM, Peterson AT, Phillips SJ, Richardson K, Scachetti-Pereira R, Schapire RE, Soberon J, Williams S, Wisz MS, Zimmermann NE: Novel methods improve prediction of species' distributions from occurrence data. Ecography 2006, 29:129-151.

48. Friedman J, Hastie T, Tibshirani R: Additive logistic regression: A statistical view of boosting. Ann Stat 2000, 28:337-374.

49. Rogers DJ: Models for vectors and vector-borne diseases. Adv Parasitol 2006, 62:1-35.

50. Jensen JR: Thematic information extraction: Image classification. In Introductory Digital Image Processing: A Remote Sensing Perspective. Edited by: Clarke KC. New Jersey: Prentice Hall PTR; , 2 1996:197-256.

51. Fielding AH, Bell JF: A review of methods for the assessment of prediction errors in conservation presence/absence models. Environ Conserv 1997, 24:38-49.

52. United States Geological Survey. [http://dds.cr.usgs.gov/srtm/version2_1/ SRTM30/srtm30_documentation.pdf].

53. Hijmans RJ, Cameron SE, Parra JL, Jones PG, Jarvis A: Very high resolution interpolated climate surfaces for global land areas. Int I Climatol 2005, 25:1965-1978.

54. Global Land Biosphere Data and Information, Goddard Space Flight Centre's Distributed Active Archive Center. [http://daac.gsfc.nasa.gov/]

55. The Globcover Project. [http://www.esa.int/due/ionia/globcover], http:// postel.mediasfrance.org.

56. Scharlemann JP, Benz D, Hay SI, Purse BV, Tatem AJ, Wint GR, Rogers DJ: Global data for ecology and epidemiology: a novel algorithm for temporal Fourier processing MODIS data. Plos One 2008, 3:e1408.

57. NASA Jet Propulsion Laboratory. [http://www2.jpl.nasa.gov/srtm/].

58. MODIS (NASA). [http://modis.gsfc.nasa.gov/about].

59. Worldclim Database. [http://www.worlddlim.org].

60. Hay SI, Tatem AJ, Graham AJ, Goetz SJ, Rogers DJ: Global environmental data for mapping infectious disease distribution. Adv Parasitol 2006, 62:37-77.

61. Hay SI: An overview of remote sensing and geodesy for epidemiology and public health application. Adv Parasitol 2000, 47:1-35.

62. Myneni RB, Hall FG, Sellers PJ, Marshak AL: The Interpretation of Spectral Vegetation Indexes. leee T Geosci Remote 1995, 33:481-486.

63. Li ZL, Becker F: Feasibility of land surface temperature and emissivity determination from AVHRR data. Rem Sens Environ 1993, 43:67-85.

64. Boyd DS, Petitcolin F: Remote sensing of the terrestrial environment using middle infrared radiation (3.0-5.0 $\mu \mathrm{m})$. Int I Remote Sens 2004, 25:3343-3368.

65. Chefaoui RM, Lobo JM: Assessing the effects of pseudo-absences on predictive distribution model performance. Ecol Model 2008, 210:478-486.

66. Ferrier S, Watson G, Pearce J, Drielsma M: Extended statistical approaches to modelling spatial pattern in biodiversity in northeast New South Wales. I. Species-level modelling. Biodivers Conserv 2002, 11:2275-2307.
67. VanDerWal J, Shoo LP, Graham C, William SE: Selecting pseudo-absence data for presence-only distribution modeling: How far should you stray from what you know? Ecol Model 2009, 220:589-594.

68. IVCC. [http://www.ivcc.com/].

69. Reinert JF, Kaiser PE, Seawright JA: Analysis of the Anopheles (Anopheles) quadrimaculatus complex of sibling species (Diptera: Culicidae) using morphological, cytological, molecular, genetic, biochemical, and ecological techniques in an integrated approach. J Am Mosa Control Assoc 1997, 13(Suppl):1-102.

70. Seawright JA, Kaiser PE, Narang SK, Tennessen K, Mitchell SE: Distribution of sibling species A, B, C, and D of the Anopheles quadrimaculatus Complex. J Agric Entomol 1992, 9:289-300.

71. Coetzee M: Distribution of the African malaria vectors of the Anopheles gambiae complex. Am J Trop Med Hyg 2004, 70:103-104.

72. Achee NL, Grieco JP, Masuoka P, Andre RG, Roberts DR, Thomas J, Briceno I, King R, Rejmankova E: Use of remote sensing and geographic information systems to predict locations of Anopheles darlingi positive breeding sites within the Sibun River in Belize, Central America. J Med Entomol 2006, 43:382-392.

73. Manguin S, Roberts DR, Andre RG, Rejmankova E, Hakre S: Characterization of Anopheles darlingi (Diptera: Culicidae) larval habitats in Belize, Central America. J Med Entomol 1996, 33:205-211.

74. Nagm L, Luitgards-Moura JF, Neucamp CD, Monteiro-de-Barros FS, Honorio NA, Tsouris P, Rosa-Freitas MG: Affinity and diversity indices for anopheline immature forms. Rev Inst Med Trop Sao Paulo 2007, 49:309-316.

75. Rozendaal JA: Relations between Anopheles darlingi breeding habitats, rainfall, river level and malaria transmission rates in the rain forest of Suriname. Med Vet Entomol 1992, 6:16-22.

76. Savage HM, Rejmankova E, Arredondo-Jiménez Jl, Roberts DR Rodriguez MH: Limnological and botanical characterization of larval habitats for two primary malarial vectors, Anopheles albimanus and Anopheles pseudopunctipennis, in coastal areas of Chiapas State, Mexico. J Am Mosa Control Assoc 1990, 6:612-620.

77. Mekuria Y, Tidwell MA, Williams DC, Mandeville JD: Bionomic studies of the Anopheles mosquitoes of Dajabon, Dominican Republic. J Am Mosa Control Assoc 1990, 6:651-657.

78. Vazquez-Martinez MG, Rodriguez MH, Arredondo-Jimenez Jl, MendezSanchez JD, Bond-Compean JG, Gold-Morgan M: Cyanobacteria associated with Anopheles albimanus (Diptera: Culicidae) larval habitats in southern Mexico. J Med Entomol 2002, 39:825-832.

79. Rejmankova E, Roberts DR, Manguin S, Pope KO, Komarek J, Post RA: Anopheles albimanus (Diptera: Culicidae) and cyanobacteria: an example of larval habitat selection. Environ Entomol 1996, 25:1058-1067.

80. Olano V, Carrasquilla G, Mendez F: Transmision de la malaria urbana en Buenaventura, Colombia: aspectos entomologicos. Rev Panam Salud Publica 1997, 1:287-294

81. Rodriguez AD, Rodriguez MH, Meza RA, Hernandez JE, Rejmankova E, Savage HM, Roberts DR, Pope KO, Legters L: Dynamics of population densities and vegetation associations of Anopheles albimanus larvae in a coastal area of southern Chiapas, Mexico. J Am Mosq Control Assoc 1993, 9:46-58.

82. Santamarina Mijares A, Perez Pacheco R: Reduction of mosquito larval densities in natural sites after introduction of Romanomermis culicivorax (Nematoda: Mermithidae) in Cuba. J Med Entomol 1997, 34:1-4.

83. Arredondo-Jimenez Jl, Valdez-Delgado KM: Effect of Novaluron (Rimon 10 $\mathrm{EC}$ ) on the mosquitoes Anopheles albimanus, Anopheles pseudopunctipennis, Aedes aegypti, Aedes albopictus and Culex quinquefasciatus from Chiapas, Mexico. Med Vet Entomol 2006, 20:377-387.

84. Hernandez JE, Epstein LD, Rodriguez $M H$, Rodriguez $A D$, Rejmankova $E_{\text {, }}$ Roberts DR: Use of generalized regression tree models to characterize vegetation favoring Anopheles albimanus breeding. J Am Mosa Control Assoc 1997, 13:28-34.

85. Hobbs JH, Sexton JD, St Jean Y, Jacques JR: The biting and resting behavior of Anopheles albimanus in northern Haiti. J Am Mosa Control Assoc 1986, 2:150-153.

86. Solarte Y, Hurtado C, Gonzalez R, Alexander B: Man-biting activity of Anopheles (Nyssorhynchus) albimanus and An. (Kerteszia) neivai (Diptera: Culicidae) in the Pacific lowlands of Colombia. Mem Inst Oswaldo Cruz 1996, 91:141-146. 
87. Arredondo-Jimenez JI, Bown DN, Rodriguez MH, Loyola EG: Control of Anopheles albimanus mosquitos in southern Mexico by spraying their preferred indoor resting sites. Bull World Health Organ 1995, 73:329-337.

88. Arredondo-Jimenez Jl, Rodriguez MH, Bown DN, Loyola EG: Indoor lowvolume insecticide spray for the control of Anopheles albimanus in southern Mexico. Village-scale trials of bendiocarb, deltamethrin and cyfluthrin. J Am Mosa Control Assoc 1993, 9:210-220.

89. Bown DN, Rodriguez MH, Arredondo-Jimenez Jl, Loyola EG, Rodriguez MC: Intradomiciliary behavior of Anopheles albimanus on the coastal plain of southern Mexico: implications for malaria control. J Am Mosq Control Assoc 1993, 9:321-324.

90. Roberts DR, Manguin S, Rejmankova E, Andre R, Harbach RE, Vanzie E, Hakre S, Polanco J: Spatial distribution of adult Anopheles darlingi and Anopheles albimanus in relation to riparian habitats in Belize, Central America. J Vector Ecol 2002, 27:21-30.

91. Grieco JP, Achee NL, Andre RG, Roberts DR: Host feeding preferences of Anopheles species collected by manual aspiration, mechanical aspiration, and from a vehicle-mounted trap in the Toledo District, Belize, Central America. J Am Mosq Control Assoc 2002, 18:307-315.

92. Loyola EG, Gonzalez-Ceron L, Rodriguez MH, Arredondo-Jimenez Jl, Bennett S, Bown DN: Anopheles albimanus (Diptera: Culicidae) host selection patterns in three ecological areas of the coastal plains of Chiapas, southern Mexico. J Med Entomol 1993, 30:518-523.

93. Berti J, Zimmerman R, Amarista J: Spatial and temporal distribution of anopheline larvae in two malarious areas in Sucre State, Venezuela. Mem Inst Oswaldo Cruz 1993, 88:353-362.

94. Linthicum KJ: A revision of the Argyritarsis section of the subgenus Nyssorhynchus of Anopheles (Diptera: Culicidae). Mosa Syst 1988, 20:98-271.

95. da Silva-Vasconcelos A, Kato MY, Mourao EN, de Souza RT, Lacerda RN, Sibajev A, Tsouris P, Povoa MM, Momen H, Rosa-Freitas MG: Biting indices, host-seeking activity and natural infection rates of anopheline species in Boa Vista, Roraima, Brazil from 1996 to 1998. Mem Inst Oswaldo Cruz 2002, 97:151-161.

96. Almiron WR, Brewer ME: Classification of immature stage habitats of Culicidae (Diptera) collected in Cordoba, Argentina. Mem Inst Oswaldo Cruz 1996, 91:1-9.

97. Forattini OP, Kakitani I, Massad E, Marucci D: Studies on mosquitoes (Diptera: Culicidae) and anthropic environment. 3. Survey of adult stages at the rice irrigation system and the emergence of Anopheles albitarsis in south-eastern, Brazil. Rev Saude Publica 1993, 27:313-325.

98. Forattini OP, Kakitani I, Massad E, Marucci D: Studies on mosquitoes (Diptera: Culicidae) and anthropic environment. 5. Breeding of Anopheles albitarsis in flooded rice fields in south-eastern Brazil. Rev Saude Publica 1994, 28:329-331.

99. Rubio-Palis Y, Curtis CF: Biting and resting behaviour of anophelines in western Venezuela and implications for control of malaria transmission. Med Vet Entomol 1992, 6:325-334

100. Chadee DD: Indoor and outdoor host-seeking rhythms of Anopheles albitarsis (Diptera: Culicidae) in Trinidad, West Indies. J Med Entomol 1992, 29:567-569.

101. Forattini OP, Kakitani I, Massad E, Marucci D: Studies on mosquitoes (Diptera: Culicidae) and anthropic environment. 7. Behaviour of adults Nyssorhynchus anophelines with special reference to Anopheles albitarsis s.l. in south-eastern Brazil. Rev Saude Publica 1995, 29:20-26.

102. Rubio-Palis Y, Curtis CF, Gonzales C, Wirtz RA: Host choice of anopheline mosquitoes in a malaria endemic area of western Venezuela. Med Vet Entomol 1994, 8:275-280

103. Mitchell CJ, Monath TP, Sabattini MS, Cropp CB, Daffner JF, Calisher CH, Jakob WL, Christensen HA: Arbovirus investigations in Argentina, 19771980. II. Arthropod collections and virus isolations from Argentine mosquitoes. Am J Trop Med Hyg 1985, 34:945-955.

104. Povoa MM, de Souza RT, Lacerda RN, Rosa ES, Galiza D, de Souza JR, Wirtz RA, Schlichting CD, Conn JE: The importance of Anopheles albitarsis $\mathrm{E}$ and An. darlingi in human malaria transmission in Boa Vista, state of Roraima, Brazil. Mem Inst Oswaldo Cruz 2006, 101:163-168.

105. Correa TG, Ferreira JM, Riet-Correa G, Ruas JL, Schild AL, Riet-Correa F, Guimaraes A, Felippe-Bauer ML: Seasonal allergic dermatitis in sheep in southern Brazil caused by Culicoides insignis (Diptera: Ceratopogonidae). Vet Parasitol 2007, 145:181-185.
106. Grillet ME: Factors associated with distribution of Anopheles aquasalis and Anopheles oswaldoi (Diptera: Culicidae) in a malarious area, northeastern Venezuela. J Med Entomol 2000, 37:231-238.

107. Zoppi de Roa E, Gordon E, Montiel E, Delgado L, Berti J, Ramos S: Association of cyclopoid copepods with the habitat of the malaria vector Anopheles aquasalis in the peninsula of Paria, Venezuela. J Am Mosa Control Assoc 2002, 18:47-51.

108. Grillet ME, Montañez H, Berti J: Estudio biosistemático y ecológico de Anopheles aquasalis y sus implicaciones para el control de la malaria en el estado Sucre: Il. Ecología de sus criaderos. Bol Dir Malariol San Amb 1998, 38:38-46

109. Manguin S, Peyton EL, James AC, Roberts DR: Apparent changes in the abundance and distribution of Anopheles species on Grenada Island. J Am Mosa Control Assoc 1993, 9:403-407.

110. Flores-Mendoza C, Cunha RA, Rocha DS, Lourenco-de-Oliveira R: Determinacao das fontes alimentares de Anopheles aquasalis (Diptera: Culicidae) no Estado do Rio de Janeiro, Brasil, pelo teste de precipitina. Rev Saude Publica 1996, 30:129-134.

111. Xavier MM, Rebelo JM: Especies de Anopheles (Culicidae, Anophelinae) em area endemica de malaria, Maranhao, Brasil. Rev Saude Publica 1999, 33:535-541.

112. Berti J, Zimmerman R, Amarista J: Adult abundance, biting behavior and parity of Anopheles aquasalis, Curry 1932 in two malarious areas of Sucre State, Venezuela. Mem Inst Oswaldo Cruz 1993, 88:363-369.

113. Brochero HL, Rey G, Buitrago LS, Olano VA: Biting activity and breeding sites of Anopheles species in the municipality Villavicencio, Meta, Colombia. J Am Mosa Control Assoc 2005, 21:182-186.

114. Vittor AY, Pan W, Gilman RH, Tielsch J, Glass G, Shields T, SanchezLozano W, Pinedo W, Salas-Cobos E, Flores S, Patz JA: Linking deforestation to malaria in the Amazon: characterization of the breeding habitat of the principal malaria vector, Anopheles darlingi. Am J Trop Med Hyg 2009, 81:5-12.

115. Moreno JE, Rubio-Palis Y, Acevedo P: Identificación de criaderos de anofelinos en un área endémica del estado Bolívar, Venezuela. Bol Malariol San Amb 2000, 40:21-30.

116. Zimmerman RH, Galardo AK, Lounibos LP, Arruda M, Wirtz R: Bloodmeal hosts of Anopheles species (Diptera: Culicidae) in a malaria-endemic area of the Brazilian Amazon. J Med Entomol 2006, 43:947-956.

117. Moreno JE, Rubio-Palis Y, Paez E, Perez E, Sanchez V: Abundance, biting behaviour and parous rate of anopheline mosquito species in relation to malaria incidence in gold-mining areas of southern Venezuela. Med Vet Entomol 2007, 21:339-349.

118. Magris M, Rubio-Palis Y, Menares C, Villegas L: Vector bionomics and malaria transmission in the Upper Orinoco River, Southern Venezuela. Mem Inst Oswaldo Cruz 2007, 102:303-311.

119. Tadei WP, Thatcher BD, Santos JM, Scarpassa VM, Rodrigues IB, Rafael MS: Ecologic observations on anopheline vectors of malaria in the Brazilian Amazon. Am J Trop Med Hyg 1998, 59:325-335.

120. Rosa-Freitas MG, Broomfield G, Priestman A, Milligan PJ, Momen $H$, Molyneux DH: Cuticular hydrocarbons, isoenzymes and behavior of three populations of Anopheles darlingi from Brazil. J Am Mosa Control AssoC 1992, 8:357-366.

121. Charlwood JD: Biological variation in Anopheles darlingi Root. Mem Inst Oswaldo Cruz 1996, 91:391-398.

122. Lourenco-de-Oliveira R, Guimaraes AE, Arle M, da Silva TF, Castro MG, Motta MA, Deane LM: Anopheline species, some of their habits and relation to malaria in endemic areas of Rondonia State, Amazon region of Brazil. Mem Inst Oswaldo Cruz 1989, 84:501-514.

123. Girod R, Gaborit P, Carinci R, Issaly J, Fouque F: Anopheles darlingi bionomics and transmission of Plasmodium falciparum, Plasmodium vivax and Plasmodium malariae in Amerindian villages of the UpperMaroni Amazonian forest, French Guiana. Mem Inst Oswaldo Cruz 2008, 103:702-710.

124. McHugh CP: Ecology of a semi-isolated population of adult Anopheles freeborni: abundance, trophic status, parity, survivorship, gonotrophic cycle length, and host selection. Am J Trop Med Hyg 1989, 41:169-176.

125. Washino RK, Tempelis CH: Host-feeding patterns of Anopheles freeborni in the Sacramento Valley, California. J Med Entomol 1967, 4:311-314.

126. Orr BK, Resh VH: Experimental test of the influence of aquatic macrophyte cover on the survival of Anopheles larvae. J Am Mosa Control Assoc 1989, 5:579-585. 
127. Wekesa JW, Yuval B, Washino RK: Spatial distribution of adult mosquitoes (Diptera: Culicidae) in habitats associated with the rice agroecosystem of northern California. J Med Entomol 1996, 33:344-350.

128. Wekesa JW, Yuval B, Washino RK: Multiple blood feeding by Anopheles freeborni and Culex tarsalis (Diptera: Culicidae): spatial and temporal variation. J Med Entomol 1997, 34:219-225.

129. Conn JE, Wilkerson RC, Segura MN, de Souza RT, Schlichting CD, Wirtz RA, Povoa MM: Emergence of a new neotropical malaria vector facilitated by human migration and changes in land use. Am J Trop Med Hyg 2002, 66:18-22.

130. Quinones ML, Suarez MF: Indoor resting heights of some anophelines in Colombia. J Am Mosq Control Assoc 1990, 6:602-604.

131. Lourenco-de-Oliveira R, Luz SL: Simian malaria at two sites in the Brazilian Amazon. II. Vertical distribution and frequency of anopheline species inside and outside the forest. Mem Inst Oswaldo Cruz 1996, 91:687-694

132. Rubio-Palis Y, Wilkerson R, Guzman H: Morphological characters of adult Anopheles (Nyssorhynchus) marajoara in Venezuela. J Am Mosq Control Assoc 2003, 19:107-114.

133. Motoki MT, Wilkerson RC, Sallum MA: The Anopheles albitarsis complex with the recognition of Anopheles oryzalimnetes Wilkerson and Motoki, n. sp. and Anopheles janconnae Wilkerson and Sallum, n. sp. (Diptera: Culicidae). Mem Inst Oswaldo Cruz 2009, 104:823-850.

134. Conn J: A genetic study of the malaria vector Anopheles nuneztovari from western Venezuela. J Am Mosa Control Assoc 1990, 6:400-405.

135. Fritz GN, Conn J, Cockburn A, Seawright J: Sequence analysis of the ribosomal DNA internal transcribed spacer 2 from populations of Anopheles nuneztovari (Diptera: Culicidae). Mol Biol Evol 1994, 11:406-416.

136. Sierra DM, Velez ID, Linton YM: Malaria vector Anopheles (Nyssorhynchus) nuneztovari comprises one genetic species in colombia based on homogeneity of nuclear ITS2 rDNA. J Med Entomol 2004, 41:302-307.

137. Calado DC, Foster PG, Bergo ES, dos Santos CL, Galardo AK, Sallum MA: Resurrection of Anopheles goeldii from synonymy with Anopheles nuneztovari (Diptera: Culicidae) and a new record for Anopheles dunhami in the Brazilian Amazon. Mem Inst Oswaldo Cruz 2008, 103:791-799.

138. Moreno J, Rubio-Palis Y, Sánchez V, Mariani D: Primer registro de Anopheles (Nyssorhynchus) nuneztovari Gabaldón,1940 (Diptera: Culicidae) en el estado Bolívar, Venezuela y sus implicaciones ecoepidemiológicas. Entomotropica 2004, 19:55-58.

139. Brochero H, Pareja PX, Ortiz G, Olano VA: Sitios de cría y actividad de picadura de especies de Anopheles en el municipio de Cimitarra, Santander, Colombia. Biomedica 2006, 26:269-277.

140. Rojas E, Brown E, Rosas C, Scorza JV: Populations of larvae of Anopheles spp. in natural breeding sites in western Venezuela, an area of refractory malaria. Rev Saude Publica 1992, 26:336-342.

141. Tadei WP, Dutary Thatcher B: Malaria vectors in the Brazilian amazon: Anopheles of the subgenus Nyssorhynchus. Rev Inst Med Trop Sao Paulo 2000, 42:87-94.

142. Elliott $R$ : The influence of vector behavior on malaria transmission. Am J Trop Med Hyg 1972, 21:755-763.

143. Conn J, Puertas YR, Seawright JA: A new cytotype of Anopheles nuneztovari from western Venezuela and Colombia. J Am Mosa Control Assoc 1993, 9:294-301.

144. Povoa M, Wirtz R, Lacerda R, Miles M, Warhurst D: Malaria vectors in the municipality of Serra do Navio, State of Amapa, Amazon Region, Brazil. Mem Inst Oswaldo Cruz 2001, 96:179-184.

145. Galardo AK, Arruda M, D'Almeida Couto AA, Wirtz R, Lounibos LP, Zimmerman RH: Malaria vector incrimination in three rural riverine villages in the Brazilian Amazon. Am J Trop Med Hyg 2007, 76:461-469.

146. Coetzee M, Estrada-Franco JG, Wunderlich CA, Hunt RH: Cytogenetic evidence for a species complex within Anopheles pseudopunctipennis Theobald (Diptera: Culicidae). Am J Trop Med Hyg 1999, 60:649-653.

147. Estrada-Franco JG, Ma MC, Lanzaro GC, Gwadz R, Galvan-Sanchez C, Cespedes JL, Vargas-Sagarnaga R, Rodriguez R: Genetic evidence of a species complex in Anopheles pseudopunctipennis sensu lato. Bull Pan Am Health Organ 1993, 27:26-31.

148. Manguin S, Roberts DR, Peyton EL, Fernandez-Salas I, Barreto M, Fernandez Loayza R, Elgueta Spinola R, Martinez Granaou R, Rodriguez MH: Biochemical systematics and population genetic structure of Anopheles pseudopunctipennis, vector of malaria in Central and South America. Am J Trop Med Hyg 1995, 53:362-377.
149. Manguin S, Roberts DR, Peyton EL, Rejmankova E, Pecor J: Characterization of Anopheles pseudopunctipennis larval habitats. J Am Mosa Control Assoc 1996, 12:619-626.

150. Root FM, Andrews J: Malaria and anopheline survey of Granada, B. W. I. Am J Hyg 1938, 27:549-579.

151. Bond JG, Rojas JC, Arredondo-Jimenez Jl, Quiroz-Martinez H, Valle J, Williams T: Population control of the malaria vector Anopheles pseudopunctipennis by habitat manipulation. Proc Biol Sci 2004, 271:2161-2169.

152. Fernandez-Salas I, Roberts DR, Rodriguez MH, Marina-Fernandez CF: Bionomics of larval populations of Anopheles pseudopunctipennis in the Tapachula foothills area, southern Mexico. J Am Mosa Control Assoc 1994, 10:477-486.

153. Bond JG, Novelo-Gutierrez R, Ulloa A, Rojas JC, Quiroz-Martinez H, Williams T: Diversity, abundance, and disturbance response of Odonata associated with breeding sites of Anopheles pseudopunctipennis (Diptera: Culicidae) in southern Mexico. Environ Entomol 2006, 35:1561-1568.

154. Bond JG, Quiroz-Martinez H, Rojas JC, Valle J, Ulloa A, Williams T: Impact of environmental manipulation for Anopheles pseudopunctipennis Theobald control on aquatic insect communities in southern Mexico. I Vector Ecol 2007, 32:41-53.

155. Fernandez-Salas I, Rodriguez MH, Roberts DR, Rodriguez MC, Wirtz RA: Bionomics of adult Anopheles pseudopunctipennis (Diptera: Culicidae) in the Tapachula foothills area of southern Mexico. J Med Entomol 1994, 31:663-670.

156. Fernandez-Salas I, Roberts DR, Rodriguez MH, Rodriguez Mdel C, MarinaFernandez CF: Host selection patterns of Anopheles pseudopunctipennis under insecticide spraying situations in southern Mexico. J Am Mosq Control Assoc 1993, 9:375-384

157. Lardeux F, Loayza P, Bouchite B, Chavez T: Host choice and human blood index of Anopheles pseudopunctipennis in a village of the Andean valleys of Bolivia. Malar J 2007, 6:8.

158. Casas M, Bown DN, Rodriguez MH: Intradomicillary pre- and postfeeding behavior of Anopheles pseudopunctipennis of southern Mexico: implications for malaria control. J Am Mosq Control Assoc 1994, 10:348-354.

159. Loyola EG, Rodriguez MH, Gonzalez L, Arredondo Jl, Bown DN, Vaca MA: Effect of indoor residual spraying of DDT and bendiocarb on the feeding patterns of Anopheles pseudopunctipennis in Mexico. J Am Mosq Control Assoc 1990, 6:635-640.

160. Bassi DG, Weathersbee AA, Meisch MV, Inman A: Efficacy of Duplex ${ }^{\oplus}$ and Vectobac $^{\ominus}$ against Psorophora columbiae and Anopheles quadrimaculatus larvae in Arkansas ricefields. J Am Mosa Control Assoc 1989, 5:264-266.

161. McLaughlin RE, Vidrine MF, Willis OR: Incidence of patent infections of Anopheles quadrimaculatus (Diptera: Culicidae) larvae by Parathelohania anophelis (Protozoa: Microsporida) in rice fields in southwestern Louisiana. J Invertebr Pathol 1988, 51:172-174.

162. McLaughlin RE, Vidrine MF, Willis OR: Distribution of Anopheles quadrimaculatus and An. crucians larvae within rice field habitats in southwestern Louisiana. J Am Mosa Control Assoc 1987, 3:574-578.

163. Lacey LA, Inman A: Efficacy of granular formulations of Bacillus thuringiensis $(\mathrm{H}-14)$ for the control of Anopheles larvae in rice fields. J Am Mosa Control Assoc 1985, 1:38-42.

164. Kottkamp WB, Meisch MV: Efficacy of BAY SIR 8514, an insect growth regulator, against Psorophora columbiae and Anopheles quadrimaculatus in small plot and field trials. J Am Mosa Control Assoc 1985, 1:17-19.

165. Dennett JA, Meek CL, Meisch MV: Efficacy of VectoLex ${ }^{\circledast}$ WDG against Anopheles quadrimaculatus and Psorophora columbiae larvae in Arkansas and Mississippi rice. J Am Mosa Control Assoc 2001, 17:231-237.

166. Marten GG, Nguyen M, Ngo G: Copepod predation on Anopheles quadrimaculatus larvae in rice fields. J Vector Ecol 2000, 25:1-6.

167. Smith TW, Walker ED, Kaufman MG: Bacterial density and survey of cultivable heterotrophs in the surface water of a freshwater marsh habitat of Anopheles quadrimaculatus larvae (Diptera: Culicidae). J Am Mosa Control Assoc 1998, 14:72-77.

168. Jensen T, Cockburn AF, Kaiser PE, Barnard DR: Human blood-feeding rates among sympatric sibling species of Anopheles quadrimaculatus mosquitoes in northern Florida. Am J Trop Med Hyg 1996, 54:523-525.

169. Walker ED, Merritt RW: Bacterial enrichment in the surface microlayer of an Anopheles quadrimaculatus (Diptera: Culicidae) larval habitat. J Med Entomol 1993, 30:1050-1052. 
170. Robertson LC, Prior S, Apperson CS, Irby WS: Bionomics of Anopheles quadrimaculatus and Culex erraticus (Diptera: Culicidae) in the Falls Lake basin, North Carolina: seasonal changes in abundance and gonotrophic status, and host-feeding patterns. J Med Entomol 1993, 30:689-698.

171. Reinert JF, White SE, Willis OR: Immatures of Anopheles quadrimaculatus s. s. collected from atypical habitats. J Am Mosa Control Assoc 2003, 19:89-90.

172. Carlson DB, Vigliano RR, Wolfe GL: Distribution of mosquitoes in different wastewater stages of secondarily treated domestic effluent and untreated citrus washwater. J Am Mosq Control Assoc 1986, 2:516-521.

173. Kaiser PE, Narang SK, Seawright JA, Kline DL: A new member of the Anopheles quadrimaculatus complex, species C. J Am Mosq Control AssoC 1988, 4:494-499.

174. Kaiser PE, Mitchell SE, Lanzaro GC, Seawright JA: Hybridization of laboratory strains of sibling species A and B of Anopheles quadrimaculatus. J Am Mosa Control Assoc 1988, 4:34-38.

175. Forattini OP, Kakitani I, Marques GR, de Brito M: Formas imaturas de anofelíneos em recipientes artificiais. Rev Saude Publica 1998, 32:189-191.

176. Marques GR, Forattini OP: Encontro de imaturos de Anopheles cruzii em bromélias de área urbana, litoral de São Paulo. Rev Saude Publica 2009, 43:369-372.

177. Wilkerson R, Peyton EL: The Brazilian malaria vector Anopheles (Kerteszia) cruzii: life stages and biology (Diptera: Culicidae). Mosq Syst 1991, 23:110-122.

178. Gutiérrez LA, Naranjo N, Jaramillo LM, Muskus C, Luckhart S, Conn JE, Correa MM: Natural infectivity of Anopheles species from the Pacific and Atlantic Regions of Colombia. Acta Trop 2008, 107:99-105.

179. Astaiza R, Murillo C, Fajardo P: Biologia de Anopheles (Kerteszia) neivai $\mathrm{H}$. D. \& K., 1913 (Diptera: Culicidae) en la costa Pacifica de Colombia. II. Fluctuacion de la poblacion adulta. Rev Saude Publica 1988, 222:101-108.

180. Rubio-Palis $Y$ : Vector biology and malaria transmission in western Venezuela. University of London 1991.

181. Benítez JA, Rodríguez A, Sojo M, Lobo H, Villegas C, Oviedo L, Brown E: Descripción de un brote epidémico de malaria de altura en un área originalmente sin malaria del estado Trujillo, Venezuela. Bol Malariol San Amb 2004, 44:93-99.

182. Achee NL, Grieco JP, Andre RG, Rejmankova E, Roberts DR: A mark releaserecapture study to define the flight behaviors of Anopheles vestitipennis and Anopheles albimanus in Belize, Central America. J Am Mosa Control Assoc 2007, 23:276-282.

183. Achee NL, Korves CT, Bangs MJ, Rejmankova E, Lege M, Curtin D, Lenares $H$, Alonzo $Y$, Andre RG, Roberts DR: Plasmodium vivax polymorphs and Plasmodium falciparum circumsporozoite proteins in Anopheles (Diptera: Culicidae) from Belize, Central America. J Vector Ecol 2000, 25:203-211.

184. Grieco JP, Achee NL, Roberts DR, Andre RG: Comparative susceptibility of three species of Anopheles from Belize, Central America, to Plasmodium falciparum (NF-54). J Am Mosa Control Assoc 2005, 21:279-290.

185. Roberts DR, Chan O, Pecor J, Rejmankova E, Manguin S, Polanco J, Legters LJ: Preliminary observations on the changing roles of malaria vectors in southern Belize. J Am Mosa Control Assoc 1993, 9:456-459.

186. Loyola EG, Arredondo Jl, Rodriguez MH, Brown DN, Vaca-Marin MA: Anopheles vestitipennis, the probable vector of Plasmodium vivax in the Lacandon forest of Chiapas, Mexico. Trans R Soc Trop Med Hyg 1991 85:171-174.

187. Diéguez L, Rodriguez R, Atienzar E, Manso O, Basulto P, Góngora N: Observaciones entomológicas en un brote de paludismo durante la etapa de vigilancia intensiva en Albaisa, Camaguey. Rev Cubana Med Trop 2002, 54:118-126.

188. de Oliveira-Ferreira J, Lourenco-de-Oliveira R, Teva A, Deane LM, DanielRibeiro CT: Natural malaria infections in anophelines in Rondonia State, Brazilian Amazon. Am J Trop Med Hyg 1990, 43:6-10.

189. Branquinho MS, Lagos CB, Rocha RM, Natal D, Barata JM, Cochrane AH, Nardin E, Nussenzweig RS, Kloetzel JK: Anophelines in the state of Acre, Brazil, infected with Plasmodium falciparum, $P$. vivax, the variant $P$. vivax VK247 and P. malariae. Trans R Soc Trop Med Hyg 1993, 87:391-394.

190. de Arruda M, Carvalho MB, Nussenzweig RS, Maracic M, Ferreira AW, Cochrane $\mathrm{AH}$ : Potential vectors of malaria and their different susceptibility to Plasmodium falciparum and Plasmodium vivax in northern Brazil identified by immunoassay. Am J Trop Med Hyg 1986, 35:873-881.
191. Quiñones ML, Ruiz F, Calle DA, Harbach RE, Erazo HF, Linton YM: Incrimination of Anopheles (Nyssorhynchus) rangeli and An. (Nys.) oswaldoi as natural vectors of Plasmodium vivax in Southern Colombia. Mem Inst Oswaldo Cruz 2006, 101:617-623.

192. Hayes J, Calderón G, Falcón R, Zambrano V: Newly incriminated anopheline vectors of human malaria parasites in Junin Department, Peru. J Am Mosa Control Assoc 1987, 3:418-422.

193. Rubio-Palis Y, Wirtz RA, Curtis CF: Malaria entomological inoculation rates in western Venezuela. Acta Trop 1992, 52:167-174.

194. Clark GG, Quiroz Martinez H: Mosquito vector control and biology in Latin America - a 15th symposium. Abstracts. J Am Mosa Control Assoc 2005, 21:412-424.

195. Moreno JE, Rubio-Palis Y, Paez E, Perez E, Sanchez V, Vaccari E: Anopheles (Anopheles) neomaculipalpus: a new malaria vector in the Amazon basin? Med Vet Entomol 2005, 19:329-332.

196. Herrera S, Suárez MF, Sánchez Gl, Quiñónes ML, Herrera M: Uso de la técnica immuno-radiométrica (IRMA) en Anopheles de Colombia para la identificación de esporozoites de Plasmodium. Colomb Med 1987, 18:2-6.

197. MAP. [http://www.map.ox.ac.uk].

198. NOAA. [http://noaasis.noaa.gov/NOAASIS/ml/avhrr.html].

199. Macdonald G: Local features of malaria. The epidemiology and control of malaria London: Oxford University Press 1957, 63-99.

200. Wilkerson RC, Gaffigan TV, Bento Lima J: Identification of species related to Anopheles (Nyssorhynchus) albitarsis by random amplified polymorphic DNA-polymerase chain reaction (Diptera: Culicidae). Mem Inst Oswaldo Cruz 1995, 90:721-732.

201. Darsie RF, Ward RA: Identification and Geographical Distribution of the Mosquitoes of North America, North of Mexico Gainsville: Univ. Press of Florida/State Univ 2005.

202. Faran ME, Linthicum KJ: A handbook of the Amazonian species of Anopheles (Nyssorhynchus) (Diptera: Culicidae). Mosq Syst 1981, 13:1-81.

203. Arredondo-Jimenez Jl, Lopez T, Rodriguez MH, Bown DN: Small scale field trials of Bacillus sphaericus (strain 2362) against anopheline and culicine mosquito larvae in southern Mexico. J Am Mosa Control Assoc 1990, 6:300-305

204. Beach RF, Cordonrosales C, Molina E, Wirtz RA: Field evaluation of an enzyme linked immunosorbent assay for estimating the sporozoite rate in Anopheles albimanus. Am J Trop Med Hyg 1992, 47:478-483.

205. Bown DN, Rodriguez MH, Arredondo-Jimenez Jl, Loyola EG, Rodriguez MC: Age structure and abundance levels in the entomological evaluation of an insecticide used in the control of Anopheles albimanus in southern Mexico. J Am Mosq Control Assoc 1991, 7:180-187.

206. Molez JF, Desenfant $P$, Jacques JR: Bio-écologie en Haïti d'Anopheles albimanus Wiedemann, 1820 (Diptera: Culicidae). Bull Soc Pathol Exot 1998, 91:334-339.

207. Perich MJ, Boobar LR, Stivers JC, Rivera LA: Field evaluation of four biorational larvicide formulations against Anopheles albimanus in Honduras. Med Vet Entomol 1990, 4:393-396.

208. Rejmankova E, Harbin-Ireland A, Lege M: Bacterial abundance in larval habitats of four species of Anopheles (Diptera: Culicidae) in Belize, Central America. J Vector Ecol 2000, 25:229-239.

209. Richards FO Jr, Flores RZ, Sexton JD, Beach RF, Mount DL, CordonRosales C, Gatica M, Klein RE: Effects of permethrin-impregnated bed nets on malaria vectors of northern Guatemala. Bull Pan Am Health Organ 1994, 28:112-121.

210. da Rocha JA, de Oliveira SB, Povoa MM, Moreira LA, Krettli AU: Malaria vectors in areas of Plasmodium falciparum epidemic transmission in the Amazon region, Brazil. Am J Trop Med Hyg 2008, 78:872-877.

211. Forattini OP, Kakitani I, Massad E, Marucci D: Studies on mosquitoes (Diptera: Culicidae) and anthropic environment. 12. Host-seeking behaviour of Anopheles albitarsis s.l. in south-eastern Brazil. Rev Saude Publica 1996, 30:299-303.

212. Berti Moser J, Gutiérrez A, Zimmerman RH: Relaciones entre tipos de hábitat, algunas variables químicas y la presencia de larvas de Anopheles aquasalis Curry y Anopheles pseudopunctipennis Theobald en un área costera del Estado Sucre, Venezuela. Entomotropica 2004, 19:79-84.

213. Carreira-Alves JR: Encontro de anofelinos do subgênero Nyssorhynchus em recipientes artificiais, Maricá, RJ, Brasil. Rev Saude Publica 2001, 35:407-408.

214. Zimmerman RH: Ecology of malaria vectors in the Americas and future direction. Mem Inst Oswaldo Cruz 1992, 87(Suppl 3):371-383. 
215. Achee NL, Grieco JP, Andre RG, Rejmankova E, Roberts DR: A mark-releaserecapture study using a novel portable hut design to define the flight behavior of Anopheles darlingi in Belize, Central America. J Am Mosa Control Assoc 2005, 21:366-379.

216. Harris AF, Matias-Arnez A, Hill N: Biting time of Anopheles darlingi in the Bolivian Amazon and implications for control of malaria. Trans $R$ Soc Trop Med Hyg 2006, 100:45-47.

217. Moore SJ, Lenglet A, Hill N: Field evaluation of three plant-based insect repellents against malaria vectors in Vaca Diez Province, the Bolivian Amazon. J Am Mosq Control Assoc 2002, 18:107-110.

218. Rejmankova E, Rubio-Palis Y, Villegas L: Larval habitats of anopheline mosquitoes in the Upper Orinoco, Venezuela. J Vector Ecol 1999, 24:130-137.

219. Vittor AY, Gilman RH, Tielsch J, Glass G, Shields T, Lozano WS, PinedoCancino V, Patz JA: The effect of deforestation on the human-biting rate of Anopheles darlingi, the primary vector of Falciparum malaria in the Peruvian Amazon. Am J Trop Med Hyg 2006, 74:3-11.

220. Voorham J: Intra-population plasticity of Anopheles darlingi's (Diptera: Culicidae) biting activity patterns in the state of Amapa, Brazil. Rev Saude Publica 2002, 36:75-80.

221. Roberts DR, Alecrim WD: Behavioral response of Anopheles darlingi to DDT-sprayed house walls in Amazonia. Bull Pan Am Health Organ 1991, 25:210-217.

222. Klein TA, Lima JB, Tang AT: Biting behavior of Anopheles mosquitoes in Costa Marques, Rondonia, Brazil. Rev Soc Bras Med Trop 1991, 24:13-20.

223. Rozendaal JA: Observations on the distribution of anophelines in Suriname with particular reference to the malaria vector Anopheles darlingi. Mem Inst Oswaldo Cruz 1990, 85:221-234.

224. Klein TA, Lima JB: Seasonal distribution and biting patterns of Anopheles mosquitoes in Costa Marques, Rondonia, Brazil. J Am Mosq Control Assoc 1990, 6:700-707.

225. Rozendaal JA: Biting and resting behavior of Anopheles darlingi in the Suriname rainforest. J Am Mosq Control Assoc 1989, 5:351-358.

226. Roberts DR, Alecrim WD, Tavares AM, Radke MG: The house-frequenting, host-seeking and resting behavior of Anopheles darlingi in southeastern Amazonas, Brazil. J Am Mosa Control Assoc 1987, 3:433-441.

227. Achee NL, Grieco JP, Rejmankova E, Andre RG, Vanzie E, Polanco J, Briceno I, King R, Roberts DR: Biting patterns and seasonal densities of Anopheles mosquitoes in the Cayo District, Belize, Central America with emphasis on Anopheles darlingi. J Vector Ecol 2006, 31:45-57.

228. de Barros FS, Honorio NA: Man biting rate seasonal variation of malaria vectors in Roraima, Brazil. Mem Inst Oswaldo Cruz 2007, 102:299-302.

229. Rodriguez M, Perez L, Caicedo JC, Prieto G, Arroyo JA, Kaur H, SuarezMutis M, de La Hoz F, Lines J, Alexander N: Composition and biting activity of Anopheles (Diptera: Culicidae) in the Amazon region of Colombia. J Med Entomol 2009, 46:307-315.

230. Mian LS, Mulla MS, Axelrod H, Chaney JD, Dhillon MS: Studies on the bioecological aspects of adult mosquitoes in the Prado Basin of southern California. J Am Mosa Control Assoc 1990, 6:64-71.

231. Casas M, Rodriguez MH, Bown DN: Peri-intradomicillary behavior in relation to host-seeking of Anopheles pseudopunctipennis in southern Mexico. J Am Mosa Control Assoc 1994, 10:355-362.

232. Casas M, Torres JL, Bown DN, Rodriguez MH, Arredondo-Jimenez J: Selective and conventional house-spraying of DDT and bendiocarb against Anopheles pseudopunctipennis in southern Mexico. J Am Mosa Control Assoc 1998, 14:410-420.

233. Lardeux FJ, Tejerina RH, Quispe V, Chavez TK: A physiological time analysis of the duration of the gonotrophic cycle of Anopheles pseudopunctipennis and its implications for malaria transmission in Bolivia. Malar J 2008, 7:141.

234. Loyola EG, Vaca MA, Bown DN, Perez E, Rodriguez MH: Comparative use of bendiocarb and DDT to control Anopheles pseudopunctipennis in a malarious area of Mexico. Med Vet Entomol 1991, 5:233-242.

235. Apperson CS, Federici BA, Tarver FR, Stewart W: Biotic and abiotic parameters associated with an epizootic of Coelomomyces punctatus in a larval population of the mosquito Anopheles quadrimaculatus. J Invertebr Pathol 1992, 60:219-228.

236. Nayar JK, Knight JW, Kaiser PE, Seawright JA, Narang SK: Comparative susceptibility of species A, B and C of Anopheles quadrimaculatus complex to infection with subperiodic Brugia malayi and Brugia pahangi (Nematoda: Filarioidea). J Am Mosa Control Assoc 1992, 8:61-64.
237. Wallace JR, Merritt RW: Diel feeding periodicity of larval anopheline mosquitoes on microorganisms and microinvertebrates: a spatial and temporal comparison of Anopheles quadrimaculatus (Diptera: Culicidae) diets in a Michigan pond. J Med Entomol 2004, 41:853-860.

doi:10.1186/1756-3305-3-72

Cite this article as: Sinka et al:: The dominant Anopheles vectors of human malaria in the Americas: occurrence data, distribution maps and bionomic précis. Parasites \& Vectors 2010 3:72.

\section{Submit your next manuscript to BioMed Central and take full advantage of:}

- Convenient online submission

- Thorough peer review

- No space constraints or color figure charges

- Immediate publication on acceptance

- Inclusion in PubMed, CAS, Scopus and Google Scholar

- Research which is freely available for redistribution 